\title{
Activation of the hippocampal LXR $\beta$ improves sleep-deprived cognitive impairment by inhibiting neuroinflammation
}

\section{Chen Qiu}

Fourth Military Medical University: Air Force Medical University

\section{Min Wang}

Fourth Military Medical University: Air Force Medical University

\section{Wen Yu}

Fourth Military Medical University: Air Force Medical University

\section{Zheng Rong}

Fourth Military Medical University: Air Force Medical University

\section{He-Sheng Zheng}

Fourth Military Medical University: Air Force Medical University

\section{Ting Sun}

Fourth Military Medical University: Air Force Medical University

\section{Shui-Bing Liu}

Fourth Military Medical University: Air Force Medical University

\section{Ming-Gao Zhao}

Fourth Military Medical University: Air Force Medical University

Yu-Mei Wu ( $\nabla$ yumeiwu@fmmu.edu.cn )

Fourth Military Medical University https://orcid.org/0000-0002-8524-1254

\section{Research Article}

Keywords: Liver X receptors, Sleep deprivation, Cognitive impairment, High mobility group box1 (HMGB1), Microglia, Neuroinflammation

Posted Date: April 22nd, 2021

DOl: https://doi.org/10.21203/rs.3.rs-435395/v1

License: (c) (1) This work is licensed under a Creative Commons Attribution 4.0 International License. Read Full License 
Version of Record: A version of this preprint was published at Molecular Neurobiology on July 19th, 2021. See the published version at https://doi.org/10.1007/s12035-021-02446-2. 


\section{Abstract}

Sleep deprivation (SD) leads to cognitive impairment due to neuroinflammation associated with impaired hippocampal neuronal plasticity and memory processes. Liver X receptors (LXRs), including LXRa and LXRß isoforms, are crucial for synaptic plasticity as well as anti-inflammation. However, the potential roles of LXRs in the pathogenesis of cognitive impairment induced by SD remain unclear. We revealed that $S D$ resulted in $L X R \beta$ reduction in hippocampus, which was associated with upregulated expression of high mobility group box1 (HMGB1)/toll-like receptor 4 (TLR4)/NF-KB p65, and knockdown of hippocampal LXR $\beta$ by shRNA (shLXRß) led to cognitive impairment. GW3965, a dual agonist for both LXRa and LXR $\beta$, ameliorated SD-induced cognitive impairment by inhibiting microglia activation, suppressing HMGB1/TLR4/NF-KB p65 pathway, and ultimately affecting the hippocampal expression of inflammatory cytokines in SD mice. LXR $\beta$ knockdown by shLXR $\beta$, abrogated GW3965-mediated the inhibition of HMGB1/TLR4/NF-kB p65 pathway, therefore abolished the cognitive improvement. Moreover, inhibition of HMGB1 by glycyrrhizin (GLY) synergistic promoted GW3965-mediated antiinflammation in activated microglia after LPS/ATP stimulation and facilitated the cognitive improvement after GW administration by activating LXRß. All the data suggested that GW3965 ameliorated impaired cognition in SD mice by suppressing HMGB1/TLR4/NF-KB p65 pathway followed LXRß activation. This study correlates a deficit of LXR $\beta$ in cognitive dysfunction in SD associated with HMGB1 inflammatory pathway in hippocampus, and LXRs may serve as a potential therapeutic target for cognitive impairment with anti-inflammation.

\section{Introduction}

Sleep benefits proper cognitive functioning, whereas sleep disruption or deprivation (SD), getting sleep less than four hours a day, leads to cognitive deficits, such as impaired attention, decision making, learning and various types of memory [1,2]. SD pervasively links to numerous neurological and psychiatric disorders [3], and the high clinical incidence of SD seriously endangers public health and brings great burden to the society [4]. Therefore, it is necessary to determine how SD impairs cognition and may thus be a target for disease treatment and/or prevention.

Hippocampus, a neural structure, is crucially involved in many cognitive functions including the formation and optimization of learning and memory, and the consolidation of declarative memory $[5,6]$, and is particularly sensitive to sleep loss. Evidence showed that SD substantially decreased the ability to induce long-term potentiation (LTP) in hippocampus [7], and also reduced the synthesis of proteins related to hippocampal neuroplasticity and impaired neurogenesis in hippocampus [8]. SD resulted in learning and memory impairments, especially when the hippocampus was required for these learning and memory processes [9]. Furthermore, the immunological and redox system was affected upon SD injury, leading to neuroinflammation and oxidative stress, and the hippocampus was rich in microglia and inflammatory factor receptors which responded to inflammatory reaction [10]. 
As the resident immune cells in the central nervous system (CNS), microglia exert critical physiological functions in regulating neuronal activity and synaptic plasticity, thus emotions can be processed and integrated correctly by pruning synapses and secreting active substances [11, 12]. Microglia were observed activated under SD stress and followed by the release of a large number of pro-inflammatory factors, leading to cognitive impairment [9]. Therefore, it is of great importance to correct microglia dysfunction and following issues to treat cognitive impairment induced by SD [13]. High mobility group box 1 (HMGB1), a pro-inflammatory cytokine, is secreted by activated microglia [14], acting as an alarm protein for neuroinflammation [15]. HMGB1 is translocated to extracellular space from nucleus and cytoplasm, resulting in the increased NF-KB nuclear translocation by binding to toll-like receptor 4 (TLR4), a specific receptor for HMGB1 expressed exclusively in microglia in the CNS [16]. Subsequently, the expression of proinflammatory cytokines is induced including interleukin-1 $\beta$ (IL-1 $\beta$ ) and tumor necrosis factor- $a$ (TNF- $\alpha$ ) [17]. It is possible to rescue the cognitive deficit by correcting the abnormal neural plasticity mediated by neuroinflammation.

Activation of liver $X$ receptor $\beta$ (LXRß), a nuclear receptor, increases hippocampal neurogenesis, regulates synaptic plasticity, and inhibits neuroinflammation. Knockout of LXR $\beta$ leads to microglia activation, supports that LXR $\beta$ is critical in neuroinflammation $[18,19]$. GW3965 (GW), a LXRs full agonist for both LXRa and LXR $\beta$ isoforms, can readily cross the blood-brain barrier to exert its specific actions in the brain [20]. Studies have proved that GW can inhibit the release of inflammatory factors in LPS-induced inflammation and improve cognitive impairment caused by Alzheimer's disease (AD) by activating LXRs $[21,22]$. However, whether GW can ameliorate SD-induced cognitive impairment and the mechanisms of LXRs involved remain unknown.

In this study, we found that the reduction of LXR $\beta$ expression was associated with SD-induced cognitive impairment. Activation of LXRß by GW alleviated SD-induced cognitive impairment through inhibiting the activation of microglia and subsequently decreased the expression levels of HMGB1, TLR4, NF-KB p65, TNF- $\alpha$ and IL-1 $\beta$ in hippocampal microglia. We demonstrated the important role of LXR $\beta$ in SD-induced cognitive impairment and presented a new therapeutic approach to mitigate SD-induced cognitive impairment by giving GW administration.

\section{Materials And Methods}

\section{Materials}

GW3965 (purity > 98\%) and glycyrrhizin (GLY, purity > 99\%) were purchased from Selleckchem (Shanghai, China) and dissolved in sterile 0.1\% DMSO to desired concentrations before use. Anti-HMGB1, anti-TLR4, anti-CD68 and anti-Histone-H3 antibodies were purchased from Proteintech (Wuhan, China). Anti-TNF-a, anti-NF-kB p65 and anti-lba-1 were purchased from Cell Signaling Technology (Danvers, MA, USA). Anti- $\beta$ actin, anti-IL-1 $\beta$, anti-LXRa and anti-LXR $\beta$ antibodies, Alexa Fluro 488 and 594 goat IgG were purchased from Abcam (Cambridge, UK, USA). Nuclear and cytoplasmic protein extraction kit was purchased from Rovntimo (Chanahai China) All cornndary antibodies conjugated with horseradish peroxidase (HRP) Loading [MathJax]/jax/output/CommonHTML/jax.js 
were purchased from Santa Cruz Biotechnology (Santa Cruz, CA, USA). Goat serum was purchased from Boster Biological Technology (Pleasanton, CA, USA). BCA Kit, M-PER Protein Extraction Buffer and enhanced chemiluminescent solution (ECL) were obtained from Pierce (Rockford, IL, USA). PVDF membrane was purchased from Merck Millipore (Billerica, MA, USA). Adeno-associated virus (AAV)encoding shRNAs for LXRa and LXRß were prepared by GenePharma (Shanghai, China). RPMI1640 cell culture medium was purchased from Biochrom (Berlin, Germany). Fetal bovine serum (FBS) was provided by Invitrogen (Carlsbad, CA, USA). ELISA kits for HMGB1, TNF- $a$ and IL-1 $\beta$ were purchased from CUSABIO (Wuhan, China). All of the other chemicals and reagents were standard commercially available biochemical quality.

\section{Animals}

Eight- to ten-week-old adult male C57BL/6 mice (weighed 20-25 g) were obtained from Experimental Animal Center of Fourth Military Medical University (Certificate No. 201000082, Grade II). The mice were kept as one group in a $380 \times 325 \times 180 \mathrm{~mm}$ plastic box made of opaque polypropylene (By-DSLH380). They were placed in a 12-h light/dark cycle environment (temperature of $24 \pm 2^{\circ} \mathrm{C}$ and humidity of $50 \%-60 \%$ ) with food and water available ad libitum. All experimental procedures were approved by the Ethics Committee of Fourth Military Medical University (Approval reference number No. KY20193145) in full accordance with the ethical guidelines of the National Institutes of Health for the care and use of laboratory animals. The animals were habituated to laboratory conditions for at least one week before testing. All the behavioral tests were taken between 8 a.m. and 6 p.m. Every effort was made to minimize the number of animals used and their suffering.

\section{Experimental procedure}

An experimenter, who was blind to the design of the experiment, pre-designated 18 mice from 1 to 18 , and a computer randomization table was used to randomly divide the numbered mice into 3 groups, Control (Ctrl), SD, SD + GW $(10 \mathrm{mg} / \mathrm{kg})$. The experimental procedure was shown in the Fig. 1a. The mice in the SD and $S D+G W$ group were deprived of sleep $20 \mathrm{~h}$ a day for $28 \mathrm{~d}$. Ctrl mice were kept in the same environment but allowed to sleep at will. After $14 \mathrm{~d}$ of SD, the mice received $\mathrm{GW}(10 \mathrm{mg} / \mathrm{kg})$ or vehicle (saline, $10 \mathrm{ml} / \mathrm{kg}$ ) by intraperitoneally (i.p.) injection, once a day for $14 \mathrm{~d}$ (from $\mathrm{d} 14$ to $\mathrm{d} 28$ ) consecutively. Drugs were given between 9 a.m. and 10 a.m. Animal learning and memory behaviors were tested at fixed time after SD. Mice from each group were sacrificed to collect the hippocampus for immunofluorescence staining and Western blot analysis after behavior tests.

\section{Sleep deprivation procedure}

The SD model was established by using the small platform water environment method [23]. SD was performed in a $50 \times 40 \times 20 \mathrm{~cm}$ water tank with several cylindrical platform $(5 \mathrm{~cm}$ in height, $3 \mathrm{~cm}$ in diameter, and $1 \mathrm{~cm}$ on the horizontal surface), and mice were free to eat and drink on the platform. The platforms were spaced at a distance of $4 \mathrm{~cm}$ so that mice could move freely from one platform to 
caused them to drop their heads to touch the water and woke up, thus keeping the mice awake and unable to sleep. Before the experiment, the mice were adapted to the platform environment for $3 \mathrm{~d}$. During the experiment, the indoor temperature was maintained at $22-25^{\circ} \mathrm{C}$, and the $40 \mathrm{~W}$ fluorescent lamp was continuously illuminated. The mice were sleep deprived for $20 \mathrm{~h}$ a day and rested for $4 \mathrm{~h}$ ( 8 a.m. to 12 a.m.).

\section{Morris water maze}

Morris water maze (MWM) was conducted as described previously [24]. The MWM consisted of a 100 cm-diameter pool with non-toxic skim milk powder as an opaque background and divided into equalsized quadrants. A circular platform (10 cm in diameter) was used as a target and the experimental water temperature was $20 \pm 2^{\circ} \mathrm{C}$. There were different special visual cues around the pool. All behavior was recorded in real time using an automatic tracking system. During the experiment, the mice were put into the water with their heads facing the wall. The entry quadrant varied but the platform location remained constant. During the training, if the animal could not find the platform within $60 \mathrm{~s}$, it was guided to the platform by the experimenter and was allowed to sit on the platform for $15 \mathrm{~s}$ before being removed. All mice were trained for $4 d$, four times a day, and their swimming paths were recorded. One hour after the final learning trail, a single $60 \mathrm{~s}$-probe trial was conducted without the platform. The latency to the target area (the previous platform location), the time spent in the target quadrant, the number of platform crossings and distance traveled were calculated. The analysis results were used to evaluate the spatial learning and memory ability of different groups of mice.

\section{Novel object recognition}

The novel object recognition (NOR) test was performed as described previously [25]. The apparatus was composed of a $25 \mathrm{~cm} \times 25 \mathrm{~cm} \times 20 \mathrm{~cm}$ soundproof device equipped with a digital camera on the roof. The cylinder and cube shared the same volume. Habituation trial was taken on the first day and mice were placed in the behavioral chamber to adapt to the new environment. In the absence of objects, each mouse acclimated to the instrument for $10 \mathrm{~min}$. Training trial was taken on the second day. The mice were placed in the instrument with two identical cylinders placed in symmetrical corners of the box and allowed to explore freely for $10 \mathrm{~min}$. Testing trial was taken on the third day. One cylinder was replaced with a cube of similar volume in the same place and the mice were allowed to explore freely for $10 \mathrm{~min}$, and the time spent exploring each object was recorded. After each phase, the objects and the box were wiped with $70 \%$ ethanol to remove the odor of the previous experimental animal. The mice's exploratory tracks were recorded by a camera fixed above the floor and analyzed with a video-tracking system. Interaction parameters were defined as contact with the object (tail only excluded) or facing the object (distance $<2 \mathrm{~cm}$ ). The time spent exploring familiar objects (TF) and the time spent exploring the novel objects (TN) were recorded. The proportion of the exploration time exploring the novel object was defined as the "discrimination index" expressed by the ratio of TN/(TF + TN). 
To knock down endogenous LXRs, shRNA-coding plasmids against LXRa (Nr1h3) and LXR $(\mathrm{Nr} 1 \mathrm{~h} 2)$ were designed according to the validated shRNA sequences [26]. The sequence of shRNA for $L X R a$ (shLXRa) was 5'-TGCCTGATGTTTCTCCTGAT-3', and that of shRNA for LXRß (shLXRß) was 5'-

GGATTCAGAAGCAGCAACAT-3'. The AAV vector was generated after cloning shRNA fragments into the AAV vector GV478. Negative control shRNA for LXRs (shNC) served as an AAV infection control.

\section{Stereotaxic surgery and microinjections}

The animals were anesthetized with sodium pentobarbital (40 mg/kg. i.p.) and were mounted on a stereotaxic apparatus (RWD68001, Shenzhen Ruiwode Life Science, China). An attenuated glass electrode (approximately $10 \mu \mathrm{m}$ in diameter) was implanted bilaterally into the hippocampus $(-1.7 \mathrm{~mm}$ anteroposterior, $\pm 1.2 \mathrm{~mm}$ lateral to the midline, and $-2.1 \mathrm{~mm}$ dorsoventral). The mice received AAV $\operatorname{shLXRa}, \operatorname{shLXR} \beta\left(1 \mu \mathrm{l}, 1 \times 10^{9} \mathrm{gc} / \mathrm{ml}\right)$ or $\operatorname{shLXRa}+\beta\left(1 \mu \mathrm{lhLXRa}\right.$ and $\left.1 \mu \mathrm{l} \operatorname{shLXR} \beta, 1 \times 10^{9} \mathrm{gc} / \mathrm{ml}\right)$ bilaterally at $0.25 \mu \mathrm{l} / \mathrm{min}$ driven by an infusion pump (Harvard Apparatus, MA). shNC served as an infection control. $21 \mathrm{~d}$ after shRNA infection, the mice were tested for learning and memory behavior.

\section{Western blot analysis}

Protein samples were harvested from mice hippocampus or cultured N9 microglial cells after various treatments. Total proteins were lysed by M-PER Protein Extraction Buffer. Nuclear and cytoplasmic proteins of hippocampus were extracted with nuclear and cytoplasmic protein extraction kit according to the manufacturer's instructions. Protein concentrations were determined using a BCA Kit. Thirty micrograms of protein per samples were run on $12 \%$ SDS-PAGE to separate target proteins, and were subsequently transferred to PVDF membrane. The membrane was blocked with $5 \%$ non-fat milk in Trisbuffered saline containing $0.1 \%$ Tween-20 for $60 \mathrm{~min}$ at room temperature. Subsequently the membranes were incubated in the primary antibody solutions respectively, overnight at $4{ }^{\circ} \mathrm{C}$. The primary antibodies included anti-LXRa (1:1,000), anti-LXRß $(1: 1,000)$, anti-HMGB1 $(1: 1,000)$, anti-TLR4 $(1: 1,000)$, anti-TNF-a $(1: 1,000)$, anti-IL-1 $\beta(1: 1,000)$, anti-NF-KB p65 $(1: 1,000)$, anti-Iba-1 $(1: 1,000)$ and anti-CD68 $(1: 1,000)$, antiHistone-H3 $(1: 2,000)$ and anti- $\beta$-actin $(1: 10,000)$ served as loading controls. After incubation with the appropriate HRP-conjugated secondary antibody, the proteins were visualized and digitized using ECL solution and Image J program.

\section{Immunohistochemistry}

The immunohistochemistry was conducted as described before [27]. The animals were anesthetized with sodium pentobarbital ( $40 \mathrm{mg} / \mathrm{kg}$. i.p.) after the behavioral studies, and perfused with PBS first and then with $4 \%$ paraformaldehyde. Brains were quickly removed and fixed overnight. After dehydration and cryoprotection in $30 \%$ sucrose at $4^{\circ} \mathrm{C}$ for $48 \mathrm{~h}$, the hippocampus segments of the frozen brain were sectioned into $30-\mu \mathrm{m}$ slices. All sections were washed with $0.1 \%$ Triton X-100 in PBS for $30 \mathrm{~min}$ and blocked in 10\% goat serum for $1 \mathrm{~h}$. Then the brain slices were incubated with anti-LXR $\beta$ antibody (1:200) and anti-lba-1 antibody (1:100) overnight at $4^{\circ} \mathrm{C}$, followed by Alexa Fluor secondary antibody incubation. 
Nuclei were counterstained with Hoechst 33258. Fluorescent signals were photographed and analyzed using confocal fluorescence microscopy (Olympus, Japan).

\section{Cell culture and treatment}

One cell line of murine microglia N9 was provided by the Shanghai Si-xin Biotechnology Ltd. (Shanghai, China) and cultured in RPMI 1640 supplemented with $10 \% \mathrm{FBS}, 100 \mathrm{IU} / \mathrm{mL}$ penicillin and $100 \mu \mathrm{g} / \mathrm{mL}$ streptomycin. The cells were cultured at $37^{\circ} \mathrm{C}$ in the presence of $5 \% \mathrm{CO} 2$ with the culture medium changed daily. Cells were pretreated with $\mathrm{GW}(0,1,10,100 \mu \mathrm{M})$ for $2 \mathrm{~h}$ and then subjected to the stimulation of LPS $(1 \mu \mathrm{g} / \mathrm{mL})$ for $4 \mathrm{~h}$ and ATP $(2.5 \mathrm{mM})$ for $0.5 \mathrm{~h}$. For the experiment utilizing shLXR $(1$ $\times 10^{9} \mathrm{gc} / \mathrm{ml}$ ), cultured microglia N9 cells were transfected with the AAV shRNAs for $24 \mathrm{~h}$ according to the manufacturer's instruction. The cells were cultured for $72 \mathrm{~h}$ after infection and then treated as before. The knockdown efficiency of shLXR $\beta$ was determined at $72 \mathrm{~h}$ after infection using Western blot analysis. For the experiment utilizing inhibitors, cells were pretreated with GLY $(50 \mu \mathrm{M})$ for $10 \mathrm{~h}$ and then treated with or without GW $(10 \mu \mathrm{M})$ for $2 \mathrm{~h}$. Then cells were stimulated with LPS $(1 \mu \mathrm{g} / \mathrm{mL})$ for $4 \mathrm{~h}$ and ATP $(2.5 \mathrm{mM})$ for $0.5 \mathrm{~h}$. Samples were collected by centrifugation for subsequent experiments.

\section{Enzyme-linked immunosorbent assay}

The supernatant of cultured N9 cells was harvested after various treatment, and subjected to enzymelinked immunosorbent assay (ELISA) to detect the changes of factors involved. The supernatant was centrifuged at $2-8^{\circ} \mathrm{C}$ for $20 \mathrm{~min}(2,000-3,000 \mathrm{rpm})$, and the supernatant was carefully collected. The contents of HMGB1, TNF- $\alpha$ and IL-1 $\beta$ in supernatant of cells were measured by the ELISA kits according to the manufactures' protocols. The absorbance value was measured with an enzyme-labeled instrument (Bio-Rad, USA). The concentrations of samples were determined by absorbance value and standard curve.

\section{Statistical analysis}

Results were presented as the mean and SEM (mean \pm SEM). Unpaired student t-test was used to test difference between the two groups. One-way ANOVA followed by a post hoc Tukey test was used to test the difference among multiple groups. In all cases, $p<0.05$ was considered statistically significant. All statistical analysis was performed using GraphPad Prism 7.03 and SPSS statistical software package version 20.0

\section{Results}

\section{The expression of LXR $\beta$ was decreased in hippocampus of SD mice}

To determine whether LXRs signaling was required in SD, the SD model was established using a small platform water environment, in which the mice were deprived of $20 \mathrm{~h}$-sleep a day for $28 \mathrm{~d}$. The expression changes of two subtypes of LXRs in hippocampus were detected after SD. Interestingly, the expression Loading [MathJax]/jax/output/CommonHTML/jax.js 
level of LXR $\beta$ in SD group was substantially decreased to $37.94 \% \pm 5.23 \%$ of $C \operatorname{trl}(p<0.001$, SD group vs. Ctrl; Fig. 1b), while the expression level of LXRa in hippocampus was not altered after SD ( $p>0.05, S D$ group vs. Ctrl; Fig. 1b). These data suggested that SD elicited the reduction of LXR $\beta$ expression in hippocampus and activation of LXRß with an agonist might alleviate SD-induced symptoms.

\section{LXRs agonist GW3965 alleviated SD-induced cognitive impairment}

Cognitive impairment is one of the most important symptoms induced by SD [28]. In this study, two classic behavioral methods, MWM and NOR were used to examine the cognitive changes of mice from each group. The experimental process was shown in Fig. 1a. As expected, SD mice spent more time to find the platform in MWM test, which was manifested as an increase in escape latency of $39.01 \pm 2.61 \mathrm{~s}$ at $d 4$, compared with Ctrl group of $10.89 \pm 2.24 \mathrm{~s}$ ( $p<0.001$ at $d 4$, SD group vs. Ctrl; Fig. 1c). The swimming distance in SD group was $14.82 \pm 7.47 \mathrm{~m}$ at $\mathrm{d} 3$ and $12.68 \pm 1.26 \mathrm{~m}$ at $\mathrm{d} 4$, while Ctrl group was $8.43 \pm 0.98 \mathrm{~m}$ at $\mathrm{d} 3$ and $3.70 \pm 0.90 \mathrm{~m}$ at $\mathrm{d} 4(\mathrm{p}<0.01$ at $\mathrm{d} 3, \mathrm{p}<0.001$ at $d 4$, SD group vs. Ctrl; Fig. 1d). This result indicated that SD mice swam longer distances than Ctrl group before finding the platform. However, the escape latency and swimming distance in GW-treated group were significantly decreased to $14.90 \pm 1.79 \mathrm{~s}$ at $\mathrm{d} 4(\mathrm{p}<0.001, \mathrm{SD}+\mathrm{GW}$ group $v s$. SD group; Fig. $1 \mathrm{c})$ and $5.69 \pm 0.58 \mathrm{~m}$ ( $\mathrm{p}$ $<0.01, S D+G W$ group $v s$. SD group; Fig. 1d). This result indicated that GW treatment ameliorated SDinduced learning impairment. During the probe trials, SD-induced impaired memory, which was manifested as a decrease in time spent in the target quadrant and platform crossings compared with Ctrl group ( $p<0.01$, SD group vs. Ctrl; Fig. 1e; $p<0.001$, SD group vs. Ctrl; Fig. 1f). However, the impaired memory was improved after GW administration ( $p<0.05$, SD + GW group vs. SD group; Fig. 1e, f). In NOR test, SD decreased the time spent on exploring the novel object. The discrimination index in SD group was $49.28 \% \pm 2.62 \%$ compared with Ctrl group of $69.05 \% \pm 2.66 \%(p<0.001$, SD group vs. Ctrl; Fig. $1 \mathrm{~g})$, indicating that SD impaired the memory of mice. As expected, GW treatment alleviated this decrease to $61.23 \% \pm 1.80 \%(p<0.01, S D+G W$ group vs. SD group; Fig. $1 g)$. Finally, to exclude the interference of locomotor activity, the locomotor abilities of different groups were analyzed and there were no differences among the three groups ( $p>0.05$; Fig. $1 \mathrm{~h}$ ). Consistent with previous studies, SD-induced cognitive impairment was observed in our study. The result also proved that activating LXRs by GW alleviated cognitive impairment after SD.

\section{Knockdown of LXRß by shRNA in hippocampus led to cognitive impairment}

It had been proved that SD led to a significant decrease of LXRß expression in hippocampus. This result suggested that hippocampal LXR $\beta$ defects might be associated with cognitive impairment. Given the lack of selective antagonists for $L X R \beta$ isoform, we used shLXR $\beta$ stereotactic injection into the hippocampus to knock down endogenous $L X R \beta$. Western blot data showed that the level of LXR $\beta$ was decreased to $33.61 \% \pm 2.28 \%$ of $\operatorname{shNC}(\mathrm{p}<0.001$, shLXRß group vs. shNC; Fig. $2 a)$ after shLXR $\beta$ transfection. WMZ result indicated that the mice in shLXR $\beta$ group spent more time and traveled longer distance to find the platform ( $p<0.001$ at $d 4$, shLXRß group vs. shNC; Fig. $2 b, c)$. Compared with the shNC group, the time snent in the tarnet nuadrant and nlatform rrossings of shLXRß group were also decreased $(p<0.05$, Loading [MathJax]/jax/output/CommonHTML/jax.js 
shLXRß group vs. shNC; Fig. $2 d, p<0.01$, shLXR 3 group $v s$. shNC; Fig. 2e). In NOR test, shLXR $\beta$ group showed a decrease in discrimination index $(p<0.01$, shLXRß group $v s$. shNC; Fig. 2f). These results indicated that reduced hippocampal LXR $\beta$ expression was associated with cognitive impairment. However, when LXRa expression was decreased to $43.38 \% \pm 3.78 \%$ of shNC $(p<0.001$, shLXRa group vs. shNC; Fig. 2a) after shLXRa infection in hippocampus, the mice showed no cognitive impairment compared with shNC mice ( $p>0.05$, shLXRa group $v s$. shNC; Fig. $2 b-f)$. Furthermore, combined shLXRa + shLXR $\beta$ infection could not lead to further reduction of $L X R a$ and $L X R \beta$ expression and more severe cognitive impairment (Fig. 2).

\section{GW3965 improved SD-induced cognitive impairment by activating LXR $\beta$ isoform}

Based on the previous results, it seemed that LXR 3 isoform played a much more important role in cognition than LXRa isoform. Since GW was a non-selective agonist for both LXRa and LXR $\beta$ subtypes, shRNA-mediated knockdown of LXRa and LXR $\beta$ in hippocampus was also used to verify the subtype of GW-mediated cognitive improvement. The SD model was set up $7 \mathrm{~d}$ after shRNA infection, and mice were treated as before (Fig. 3a). After shLXRa infection, GW could still improve SD-induced learning disabilities $(p<0.001$ at $d 4$ shLXRa $+S D+G W$ group $v s$. shLXRa + SD group; Fig. 3b, $c)$ and memory impairment ( $p$ $<0.05$ shLXRa + SD + GW group vs. shLXRa + SD group; Fig. 3h and $p<0.01$ shLXRa + SD + GW group $v s$. shLXRa + SD group; Fig. 3i). This result indicated that knockdown of hippocampal LXRa expression did not affect GW-mediated cognitive improvement in SD mice. However, the knockdown of hippocampal LXR $\beta$ prevented GW-mediated cognition improvement $(p>0.05$, shLXR $\beta+S D+G W$ group vs. shLXR $\beta+$ SD group; Fig. $3 d, e, h$, i). Furthermore, when the expression levels of $L X R a$ and $L X R \beta$ were both downregulated, $G W$ also failed to exert its effects $(p>0.05$, shLXRa $+\beta+S D+G W$ group $v s$. shLXRa $+\beta+S D$ group; Fig. $3 f-i)$. These results suggested that GW relieved SD-induced cognitive impairment by activating LXRß isoform.

\section{GW relieved SD-induced microglia activation, followed by suppressing HMGB1/TLR4/NF-KB p65 pathway in hippocampus}

It had been shown that GW improved SD-induced cognitive impairment through LXR 3 , but the mechanisms involved were still unclear. Evidence showed that knockout of LXR $\beta$ led to microglia activation, suggesting LXR $\beta$ was responsible for neuroinflammation (Colonna \& Butovsky, 2017; Dai, Tan, Wu, Warner, \& Gustafsson, 2012). In this study, we found that hippocampal microglia were activated with a decreased LXR $\beta$ expression (Extended Data Fig. 1), suggesting that the status of microglia might be related to the expression level of LXR $\beta$ upon SD injury. Besides, the presence of LXR $\beta$ in the microglia was also observed (Extended Data Fig. 2). To verify whether GW could inhibit microglia activation after SD, the status of microglia in SD + GW group was tested. As expected, activated microglia in SD mice were significantly reduced after GW administration (Fig. 4a). Western blot results also showed that SD robustly enhanced the hippocampal expression level of Iba- 1 to $235.30 \% \pm 14.22 \%$ of $\mathrm{Ctrl}(\mathrm{p}<0.001$, SD group vs. Ctrl; Fig. 4b, c), while GW treatment decreased this level to $121.30 \% \pm 5.67 \%$ of $\mathrm{Ctrl}(p<0.001, \mathrm{SD}+\mathrm{GW}$ 
group vs. SD group; Fig. 4b, c). These results confirmed that GW could mitigate microglia activation induced by SD in hippocampus.

HMGB1 is a typical damage-associated molecule involved in various neuroinflammatory and neurodegenerative diseases, and the activation of HMGB1/TLR4/NF-KB p65 pathway is highly associated with microglial activation and leads to elevated level of inflammatory factors. In this study, we found that the hippocampal expression of HMGB1 and TLR4 was increased to $151.00 \% \pm 7.50 \%$ and $205.30 \% \pm 7.70 \%$ of Ctrl after SD ( $p<0.001$, SD group vs. Ctrl; Fig. 5a, b, d, e). Besides, SD also increased nuclear level of NF-KB p65 and decreased cytoplasmic NF-kB p65 expression correspondingly in hippocampus ( $p<0.001$, SD group vs. Ctrl; Fig. 5g-i), leading to elevated TNF- $\alpha$ and IL-1 $\beta(p<0.001$, SD group vs. Ctrl; Fig. 5c, f). However, GW treatment could significantly reduce the protein levels of HMGB1, TLR4, nuclear NF-kB p65, TNF- $a$ and IL-1 $\beta$ (SD + GW group vs. SD group; Fig. 5a-i), suggesting that GW could ameliorate neuroinflammation in hippocampus by inhibiting the HMGB1 pathway.

\section{GW suppressed HMGB1/TLR4/NF-KB p65 pathway in activated microglia by activating LXR $\beta$}

The inhibitory effects of GW on HMGB1 pathway in activated microglia were further confirmed in cultured murine $\mathrm{N} 9$ microglia in vitro. GW pre-treatment dose-dependently decreased the induction of Iba-1 and CD68, a marker of activated microglia, induced by LPS/ATP stimulation shown in extended data Fig. 3. On account of no obvious differences between $10 \mu \mathrm{M}$ and $100 \mu \mathrm{M}$ group, $10 \mu \mathrm{M}$ of GW was used in the following trials.

To verify whether the inhibitory effect of GW was through $L X R \beta$, shLXR $\beta$ was further applied in cultured N9 microglia. Western blot data showed that the expression of LXRß was successfully down-regulated to $35.92 \% \pm 4.45 \%$ of shNC after shLXR 3 infection for $3 d(p<0.001$, shLXR 3 group vs. shNC; Fig. $6 a)$.

LPS/ATP stimulation robustly enhanced the expression levels of HMGB1, TLR4, nuclear NF-KB p65, TNF$a$ and IL-1 $\beta$, which were relieved by the pre-treatment of GW as shown in Fig. 6 . However, the proteins in HMGB1 pathway were increased in shLXR + LPS/ATP + GW group compared with shNC + LPS/ATP + GW group, and showed little difference compared with shLXR + LPS/ATP group (Fig. 6b-h). The results indicated that GW inhibited HMGB1/TLR4/NF-KB p65 pathway in activated microglia due to the presence of LXRß.

HMGB1, TNF- $a$ and IL-1 $\beta$ were released from cells to exert the pro-inflammatory roles, then the content changes in the supernatant were also detected by ELISA assay. The ELISA data, showed in extended data figure 4, exhibited similar tendency to that of Western blot results as showed in Fig. 6 . The expression of HMGB1, TNF- $a$ and IL-1 $\beta$ in the supernatant showed little difference between shLXR $\beta+L P S / A T P+G W$ group and shLXR + LPS/ATP group, which meant GW inhibited inflammatory cytokines released from N9 microglia by activating LXRß.

Inhibition of HMGB1 facilitated the anti-inflammatory effect of GW upon LPS/ATP-induced inflammatory responses in murine $\mathrm{N} 9$ microglia 
To further confirm that HMGB1 pathway exerted an important role in GW-mediated anti-inflammatory effects, a direct HMGB1 inhibitor GLY was used [29]. The results showed that GLY (50 $\mu \mathrm{M})$ significantly inhibited nuclear translocation of NF-KB p65 upon LPS/ATP stimulation (LPS/ATP + GLY group vs. LPS/ATP group; Fig. 7a, b), followed by the expression reductions of TNF- $a$ and IL-1 $\beta$ (LPS/ATP + GLY group vs. LPS/ATP group; Fig. 7a, c) and the release reduction into the supernatants (LPS/ATP + GLY group vs. LPS/ATP group; Fig. 7d). Furthermore, GLY administration enhanced GW-mediated the reduction of nuclear NF-KB p65 upon LPS/ATP injury, as well as the release of TNF-a and IL-1 $\beta$ (LPS/ATP $+\mathrm{GW}+\mathrm{GLY}$ group vs. LPS/ATP + GW group; Fig. 7). This result indicated that GLY could promote the antiinflammatory effects of GW in activated microglia.

\section{Inhibition of HMGB1 pathway effectively alleviated SD-induced cognitive impairment and enhanced GW- mediated cognitive improvement}

Finally, the role of HMGB1 pathway in SD-induced cognitive impairment was tested. GLY $(20 \mathrm{mg} / \mathrm{kg})$ and/or GW (10 mg/kg) were injected intraperitoneally (i.p.) starting from d 14 after SD, for $14 \mathrm{~d}$ consecutively (Fig. 8a). Behavior test showed that treatment with GLY alone for $14 \mathrm{~d}$ effectively alleviated SD-induced learning and memory deficit (SD + GLY group vs. SD group; Fig. 8b-f). Furthermore, GW and GLY had synergistic effects in improving cognitive impairment, which was manifested as a tendency to increase learning and memory functions compared with using GW or GLY alone (SD + GW + GLY group vs. SD +GW or SD + GLY group; Fig. 8b-f). Collectively, all the results confirmed that HMGB1 pathway played an important role in cognitive deficit, and the inhibition of HMGB1 enhanced GW-mediated cognitive improvement after SD.

\section{Discussion}

In the present study, we found that SD led to cognitive impairment associated with the hippocampal reduction of $L X R \beta$ expression, and knockdown of $L X R \beta$ by shLXR $\beta$ also resulted in cognitive deficit. Furthermore, it was confirmed that GW-mediated cognitive improvement was through LXR $\beta$ activation, followed by the inhibition of HMGB1/TLR4/NF-KB p65 pathway in microglia both in vitro and in vivo, thus alleviated neuroinflammation. These results suggested a link between hippocampal LXR $\beta$ deficiency and cognitive impairment and proved the important roles of LXR $\beta$ activation for the treatment of cognitive impairment induced by SD. It also provided new insights into the neuroinflammatory mechanisms of cognitive impairment.

Sleep benefits neuronal recovery and plasticity, which supports brain function, and ultimately, cognition and emotion, whereas sleep loss leads to cognitive impairment, such as learning and memory deficits [30]. Interestingly, when the hippocampus is needed for these learning and memory processes, such impairments appear to occur, suggesting that this area of the brain may be particularly sensitive to the consequences of SD. SD significantly decreased the LTP in the hippocampus and impaired hippocampal neurogenesis [31]. In addition, functional magnetic resonance imaging indicated that the hippocampal antivity wac cinnifinantly lnwor aftor $S n$ than that of the normal volunteers [32]. Exploring the impact of Loading [MathJax]/jax/output/CommonHTML/jax.js

Page $12 / 28$ 
SD may find a target for disease treatment and/or prevention under all major neurological and psychiatric conditions, including AD, schizophrenia, anxiety disorders and addiction in which comorbid sleep disruption presents [3].

Available evidence suggested that neuroinflammation impaired hippocampal neuronal plasticity and memory processes induced by SD, leading to cognitive impairment (Miller \& Spencer, 2014; Navakkode, Liu, \& Soong, 2018). It is critical to investigate the mechanism involved in SD-induced neuroinflammation. LXRs, a nuclear receptor which can inhibit the expression of proinflammatory genes in immune cells, is increasingly concerned because of its critical role in regulating neuroinflammation [33,34]. In this study, we found that SD led to the reduction of hippocampal LXR $\beta$ expression, moreover, downregulation of LXRß in hippocampus by AAV shRNA resulted in significant cognitive impairment in the mice. This suggested that LXR $\beta$ deficit might be responsible for the pathogenesis of SD-induced cognitive impairment. Furthermore, this study also confirmed that GW, a non-selective LXRs agonist, improved the cognitive function of SD mice. Due to the lack of LXR $\beta$ isoform-specific agonist, GW-mediated the improvement by activating LXR $\beta$ instead of LXRa was verified using shRNA-mediated knockdown of LXRß.

Previous study had found that knockout of LXR $\beta$ led to an increase of activated microglia, which is the important mediators of neuroinflammation and the main source of pro-inflammatory cytokines $[18,19]$. Overactivated microglia produce neurotoxicity, damage neurons and are involved in a range of neurodegenerative diseases characterized by cognitive impairment such as AD, Parkinson's diseases and Huntington's diseases [35]. HMGB1, a typical damage associated molecule, has been shown to be released by hippocampal microglia upon unpredictable stress [14]. The release of HMGB1 from microglia is decreased after the inhibition of microglia activation, thus the HMGB1 protein level is restored accordingly [14]. HMGB1 serves as a risk factor for memory impairment, chronic neurodegeneration, and progression of neuroinflammation in neurodegenerative diseases [36]. Released HMGB1 binds to TLR4, a specific receptor expressed by microglia in rodents, induces the nuclear translocation of NF-KB p65 from the cytoplasm, leading to the induction of inflammatory factors $[37,38]$. Consistent with previous literatures, overactivated microglia and activation of HMGB1/TLR4/NF-KB p65 pathway upon SD injury were also observed in this study, which led to a significant increase of inflammatory factors TNF- $\alpha$ and IL$1 \beta$. Elevated levels of inflammatory factors in hippocampus led to impaired neural plasticity, thus resulted in hippocampus-dependent learning and memory impairments. Therefore, LXRß-mediated cognition improvement was due to the inhibition of microglia activation and HMGB1 pathway, which suppressed the induction of inflammatory factors upon SD stress, followed by the correction of abnormal neural plasticity.

To further confirm the cognitive improvement of GW was through inhibiting HMGB1 pathway, a direct HMGB1 inhibitor GLY was used. GLY inhibited the nuclear translocation of NF-KB p65 and the expression of TNF- $\alpha$ and IL-1 $\beta$, and played a synergistic role with GW (Fig. 7), suggesting that nuclear transfer of NFKB p65 and subsequent release of inflammatory factors were regulated by HMGB1. Besides, behavioral 
tests also showed that GLY facilitated GW-mediated improving cognitive function in SD mice (Fig. 8), suggesting that inhibition of HMGB1 improved cognitive impairment.

Collectively, activating LXR $\beta$ offered the improvement in SD-induced cognitive impairment by inhibiting hippocampal microglia activation, followed by suppressing the pathway HMGB1/TLR4/NF-KB p65, and ultimately affecting the release of inflammatory factors. Furthermore, our data validated the critical role of hippocampal HMGB1 pathway contributed to cognitive impairment induced by SD. These findings provided new mechanism and target for the treatment with cognitive impairment induced by SD.

\section{Declarations}

\section{Ethics approval}

All experimental procedures were approved by the Ethics Committee of Fourth Military Medical University (Approval reference number No. KY20193145) in full accordance with the ethical guidelines of the National Institutes of Health for the care and use of laboratory animals.

\section{Consent for publication}

Written informed consent for publication was obtained from all participants.

\section{Availability of data and materials}

Datasets from this study are available from the corresponding author on reasonable request.

\section{Competing interests}

The authors declare that they have no competing interests.

\section{Funding}

This study was funded by National Natural Science Foundation of China (No. 82071515), Key International Cooperation Projects of Shaanxi Province (No. 2020KWZ-021), Military Medicine Promotion Projects (No. 2018jsts09) for Prof Wu, and Research Foundation from Social Development Science and Technology Project of Shaanxi Province for Mr Wang (No. 2020JQ-464).

\section{Author's contribution}

Chen Qiu and Min Wang wrote the manuscript. Wen Yu and Zheng Rong developed the model and performed part of the behavior tests. He-Sheng Zheng and Ting Sun participated in the Western blot and ELISA analysis. Min Wang was responsible for immunohistochemical experiments. Chen Qiu was responsible for cell culture, stereotaxic surgery and microinjections. Shui-Bing Liu and Ming-Gao Zhao analyzed the data. Chen Qiu and Yu-Mei Wu designed and supervised whole experiments. All authors 
The authors will thank Wen-Ju Wang for his great help in analyzing the data.

\section{References}

1. Sheth BR. Sleep's Role in Human Spatial Learning Commentary on Nguyen et al. Overnight sleep enhances hippocampus-dependent aspects of spatial memory. SLEEP 2013;36:1051-1057. Sleep. 2013;36(7):971-2.

2. Patti CL, Zanin KA, Sanday L, Kameda SR, Fernandes-Santos L, Fernandes HA, et al. Effects of Sleep Deprivation on Memory in Mice: Role of State-Dependent Learning. Sleep. 2010;33(12):1669-79.

3. Wulff K, Gatti S, Wettstein JG, Foster RG. Sleep and circadian rhythm disruption in psychiatric and neurodegenerative disease. Nat Rev Neurosci. 2010;11(8):589-99.

4. Lowe CJ, Safati A, Hall PA. The neurocognitive consequences of sleep restriction: A meta-analytic review. Neuroscience and biobehavioral reviews. 2017;80:586-604.

5. Hainmueller T, Bartos M. Parallel emergence of stable and dynamic memory engrams in the hippocampus. Nature. 2018;558(7709):292-+.

6. Lisman J, Buzsaki G, Eichenbaum H, Nadel L, Rangananth C, Redish AD. Viewpoints: how the hippocampus contributes to memory, navigation and cognition. Nat Neurosci. 2017;20(11):1434-47.

7. McDermott CM, LaHoste GJ, Chen C, Musto A, Bazan NG, Magee JC. Sleep deprivation causes behavioral, synaptic, and membrane excitability alterations in hippocampal neurons. The Journal of neuroscience : the official journal of the Society for Neuroscience. 2003;23(29):9687-95.

8. Fernandes C, Rocha NB, Rocha S, Herrera-Solis A, Salas-Pacheco J, Garcia-Garcia F, et al. Detrimental role of prolonged sleep deprivation on adult neurogenesis. Front Cell Neurosci. 2015;9:140.

9. Wadhwa M, Prabhakar A, Ray K, Roy K, Kumari P, Jha PK, et al. Inhibiting the microglia activation improves the spatial memory and adult neurogenesis in rat hippocampus during $48 \mathrm{~h}$ of sleep deprivation. J Neuroinflamm. 2017;14.

10. Bishir M, Bhat A, Essa MM, Ekpo O, Ihunwo AO, Veeraraghavan VP, et al. Sleep Deprivation and Neurological Disorders. Biomed Res Int. 2020;2020:5764017.

11. Marshall SA, McClain JA, Kelso ML, Hopkins DM, Pauly JR, Nixon K. Microglial activation is not equivalent to neuroinflammation in alcohol-induced neurodegeneration: The importance of microglia phenotype. Neurobiol Dis. 2013;54:239-51.

12. Wu YW, Dissing-Olesen L, MacVicar BA, Stevens B. Microglia: Dynamic Mediators of Synapse Development and Plasticity. Trends Immunol. 2015;36(10):605-13.

13. Miyanohara J, Kakae M, Nagayasu K, Nakagawa T, Mori Y, Arai K, et al. TRPM2 Channel Aggravates CNS Inflammation and Cognitive Impairment via Activation of Microglia in Chronic Cerebral Hypoperfusion. Journal of Neuroscience. 2018;38(14):3520-33. 
14. Wang B, Huang X, Pan X, Zhang T, Hou C, Su WJ, et al. Minocycline prevents the depressive-like behavior through inhibiting the release of HMGB1 from microglia and neurons. Brain Behav Immun. 2020;88:132-43.

15. Tsung A, Klune JR, Zhang X, Jeyabalan G, Cao Z, Peng X, et al. HMGB1 release induced by liver ischemia involves Toll-like receptor 4-dependent reactive oxygen species production and calciummediated signaling. Journal of Experimental Medicine. 2007;204(12):2913-23.

16. Liddelow SA, Guttenplan KA, Larke LEC, Bennett FC, Bohlen CJ, Schirmer L, et al. Neurotoxic reactive astrocytes are induced by activated microglia. Nature. 2017;541(7638):481-7.

17. Sun $X$, Zeng $H$, Wang Q, Yu Q, Wu J, Feng Y, et al. Glycyrrhizin ameliorates inflammatory pain by inhibiting microglial activation-mediated inflammatory response via blockage of the HMGB1-TLR4NF-kB pathway. Exp Cell Res. 2018;369(1):112-9.

18. Colonna M, Butovsky O. Microglia Function in the Central Nervous System During Health and Neurodegeneration. Annual Review of Immunology, Vol 35. 2017;35:441-68.

19. Dai YB, Tan XJ, Wu WF, Warner M, Gustafsson JA. Liver $X$ receptor beta protects dopaminergic neurons in a mouse model of Parkinson disease. Proc Natl Acad Sci U S A. 2012;109(32):13112-7.

20. Cui X, Chopp M, Zhang ZG, Li RW, Zacharek A, Landschoot-Ward J, et al. ABCA1/ApoE/HDL Pathway Mediates GW3965-Induced Neurorestoration After Stroke. Stroke. 2017;48(2):459-67.

21. Skerrett R, Malm T, Landreth G. Nuclear receptors in neurodegenerative diseases. Neurobiol Dis. 2014;72:104-16.

22. Nunomura S, Okayama Y, Matsumoto K, Hashimoto N, Endo-Umeda K, Terui T, et al. Activation of LXRs using the synthetic agonist GW3965 represses the production of pro-inflammatory cytokines by murine mast cells. Allergol Int. 2015;64:S11-S7.

23. Zhang K, Li YJ, Feng D, Zhang P, Wang YT, Li X, et al. Imbalance between TNF alpha and progranulin contributes to memory impairment and anxiety in sleep-deprived mice. Sci Rep-Uk. 2017;7.

24. Sun T, Li YJ, Tian QQ, Wu Q, Feng D, Xue Z, et al. Activation of liver X receptor beta-enhancing neurogenesis ameliorates cognitive impairment induced by chronic cerebral hypoperfusion. Exp Neurol. 2018;304:21-9.

25. Bhattacharya A, Kaphzan H, Alvarez-Dieppa AC, Murphy JP, Pierre P, Klann E. Genetic Removal of p70 S6 Kinase 1 Corrects Molecular, Synaptic, and Behavioral Phenotypes in Fragile X Syndrome Mice. Neuron. 2012;76(2):325-37.

26. Li YJ, Zhang K, Sun T, Wang J, Guo YY, Yang L, et al. Epigenetic suppression of liver X receptor beta in anterior cingulate cortex by HDAC5 drives CFA-induced chronic inflammatory pain. $J$ Neuroinflammation. 2019;16(1):132.

27. Tian DD, Wang M, Liu A, Gao MR, Qiu C, Yu W, et al. Antidepressant Effect of Paeoniflorin Is Through Inhibiting Pyroptosis CASP-11/GSDMD Pathway. Mol Neurobiol. 2021;58(2):761-76.

28. Hagewoud R, Havekes R, Novati A, Keijser JN, van der Zee EA, Meerlo P. Sleep deprivation impairs spatial working memory and reduces hippocampal AMPA receptor phosphorylation. J Sleep Res. 
29. Mollica L, De Marchis F, Spitaleri A, Dallacosta C, Pennacchini D, Zamai M, et al. Glycyrrhizin binds to high-mobility group box 1 protein and inhibits its cytokine activities. Chem Biol. 2007;14(4):431-41.

30. Diekelmann S, Born J. SLEEP The memory function of sleep. Nature Reviews Neuroscience. 2010;11(2):114-26.

31. Krause AJ, Simon EB, Mander BA, Greer SM, Saletin JM, Goldstein-Piekarski AN, et al. The sleepdeprived human brain. Nat Rev Neurosci. 2017;18(7):404-18.

32. Yoo SS, Hu PT, Gujar N, Jolesz FA, Walker MP. A deficit in the ability to form new human memories without sleep. Nat Neurosci. 2007;10(3):385-92.

33. Kang J, Rivest S. Lipid metabolism and neuroinflammation in Alzheimer's disease: a role for liver $X$ receptors. Endocr Rev. 2012;33(5):715-46.

34. Schulman IG. Liver $X$ receptors link lipid metabolism and inflammation. FEBS Lett. 2017;591(19):2978-91.

35. Ransohoff R. Microglia in Neurodegeneration: A (Re)Introduction. Neurobiol Aging. 2016;39:S21-S.

36. Fang P, Schachner M, Shen YQ. HMGB1 in development and diseases of the central nervous system. Mol Neurobiol. 2012;45(3):499-506.

37. Gao HM, Zhou H, Zhang F, Wilson BC, Kam W, Hong JS. HMGB1 acts on microglia Mac1 to mediate chronic neuroinflammation that drives progressive neurodegeneration. The Journal of neuroscience : the official journal of the Society for Neuroscience. 2011;31(3):1081-92.

38. Wang X, Grace PM, Pham MN, Cheng K, Strand KA, Smith C, et al. Rifampin inhibits Toll-like receptor 4 signaling by targeting myeloid differentiation protein 2 and attenuates neuropathic pain. FASEB journal : official publication of the Federation of American Societies for Experimental Biology. 2013;27(7):2713-22.

\section{Figures}


a

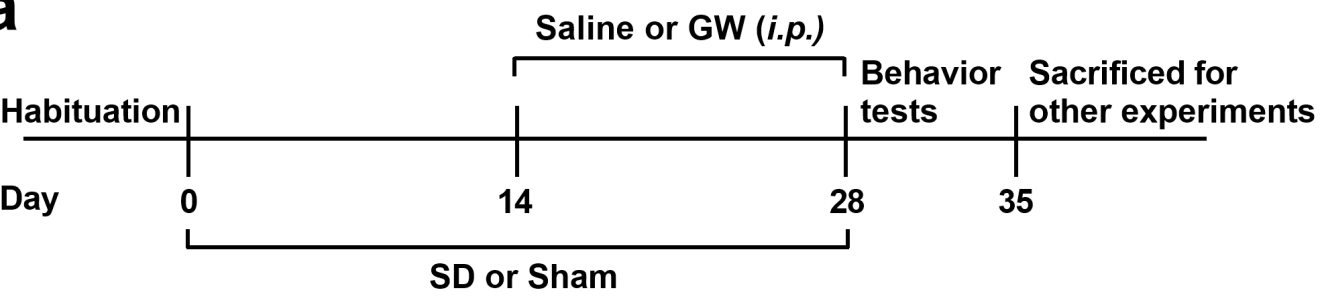

b
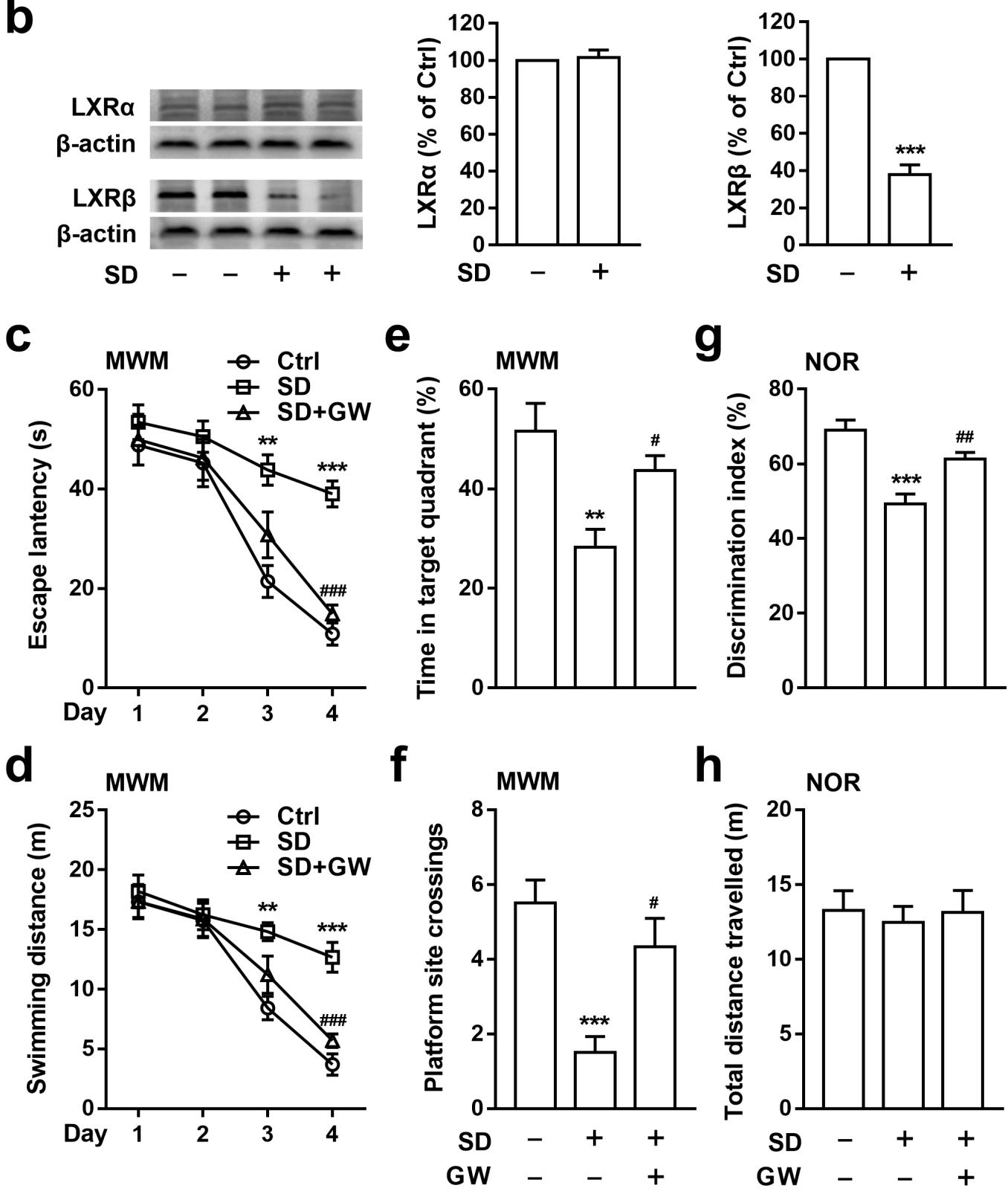

Figure 1

LXRs agonist GW3965 improved cognitive impairment induced by sleep deprivation (SD). (a) Schematic illustration of the experiment procedure. The mice were deprived of $20 \mathrm{~h}$-sleep a day for $28 \mathrm{~d}$. After SD for $14 \mathrm{~d}$, the mice received GW3965 (GW, $10 \mathrm{mg} / \mathrm{kg}$ ) or $0.9 \%$ saline by intraperitoneally (i.p.) injection, once a day for $14 \mathrm{~d}$ consecutively. (b) Western blot analysis showed no changes of LXRa and reduced LXR $\mathrm{\text {in }}$ 
impairment, which was relieved by the treatment of GW. (c) Mean daily escape latencies (time to find the hidden platform). (d) Total swimming distance during the learning phase. (e) Percentage of time spent in the target quadrant. (f) Numbers of crossing platform site spent in target quadrant. $(g, h)$ The results of novel object recognition (NOR) test. (g) Discrimination index toward a novel object. (h) Total distance travelled in NOR test. $n=6$ in each group, ${ }^{\star \star} p<0.01,{ }^{\star \star *} p<0.001$, compared with the Ctrl group; $\# p<$ $0.05, \# \# p<0.01, \# \# \# p<0.001$, compared with the SD group.

a

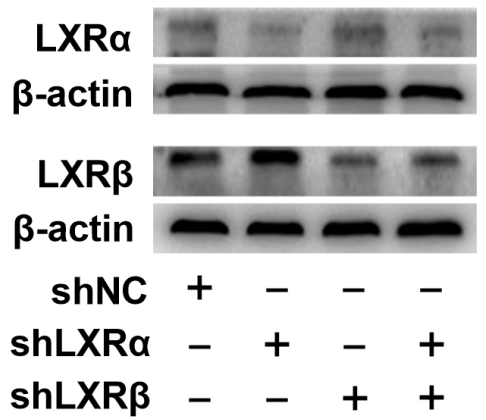

b

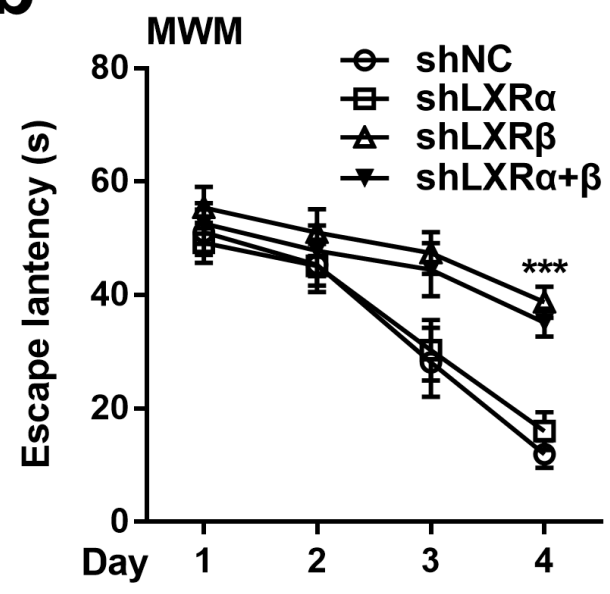

C

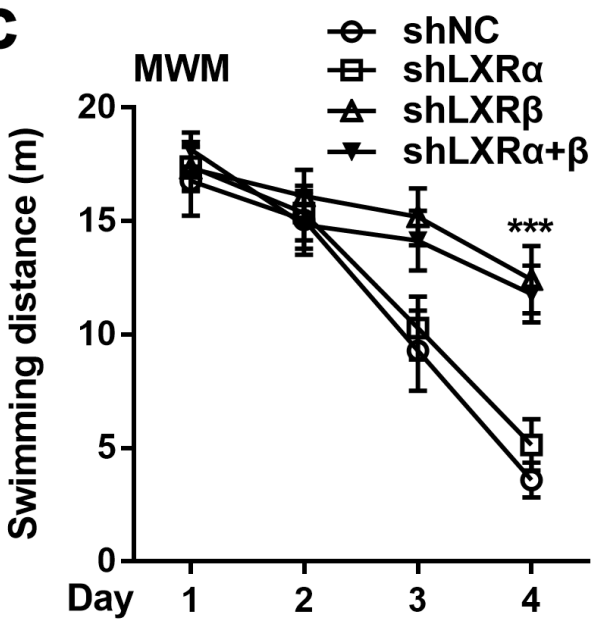

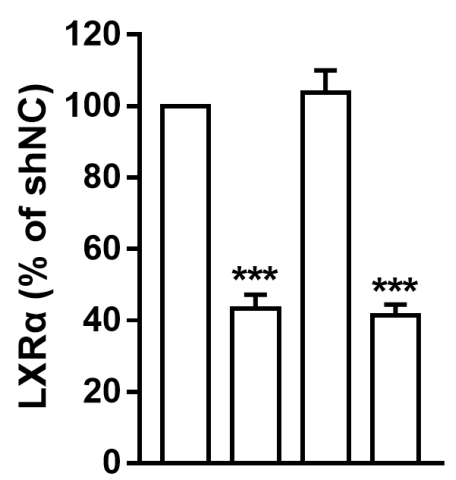

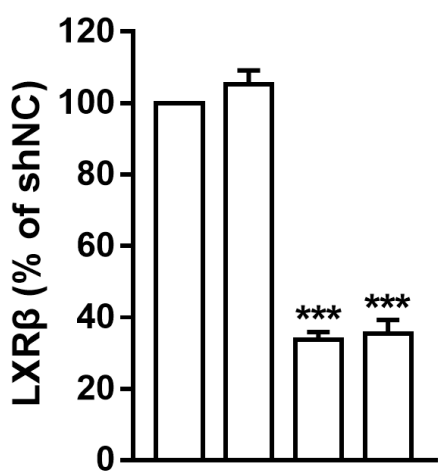

d

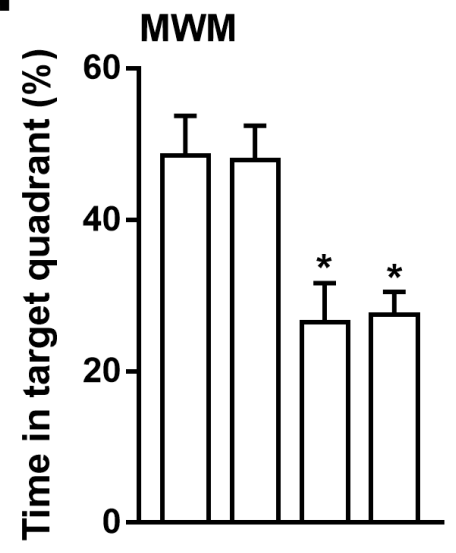

f

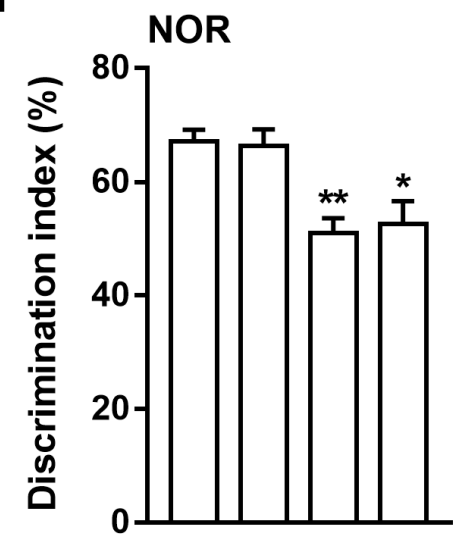

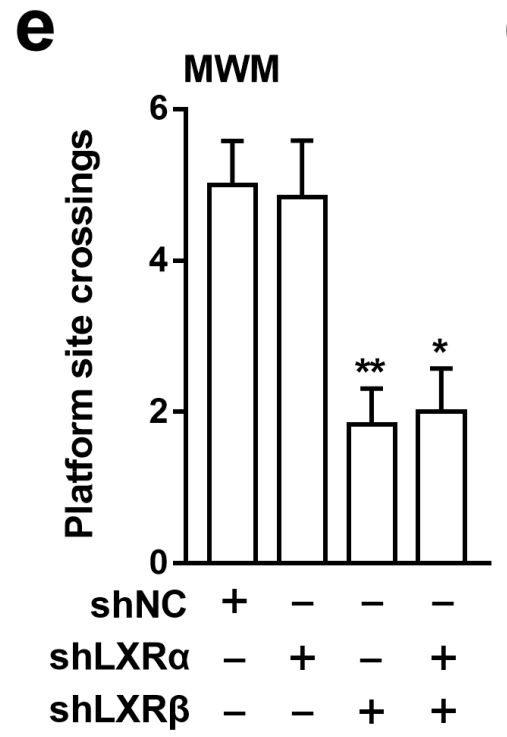

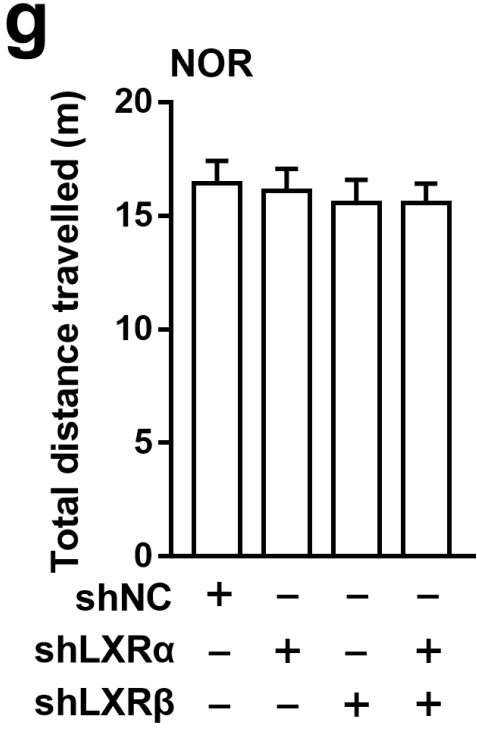


Downregulation of LXRß expression in hippocampus led to cognitive impairment. (a) The expression of $L X R a$ and $L X R \beta$ reduced after adeno-associated virus (AAV)-encoding shRNAs infection for $21 \mathrm{~d}$ in hippocampus. (b-f) The mice in shLXR $\beta$ and shLXRa+ $\beta$ groups showed cognitive impairment while the mice in shLXRa group showed no behavioral deficits after shRNA infection for $21 \mathrm{~d}$. (b) Mean daily time to find the hidden platform. (c) Total swimming distance during the learning phase. (d) Percentage of time spent in the target quadrant. (e) Numbers of crossing platform site spent in target quadrant. (f) Discrimination index toward a novel object. (g) There was no significant difference in the locomotor ability of mice in each group. $n=6$ in each group, ${ }^{*} p<0.05, * \star p<0.01, * \star \star p<0.001$, compared with the shNC group. 
a
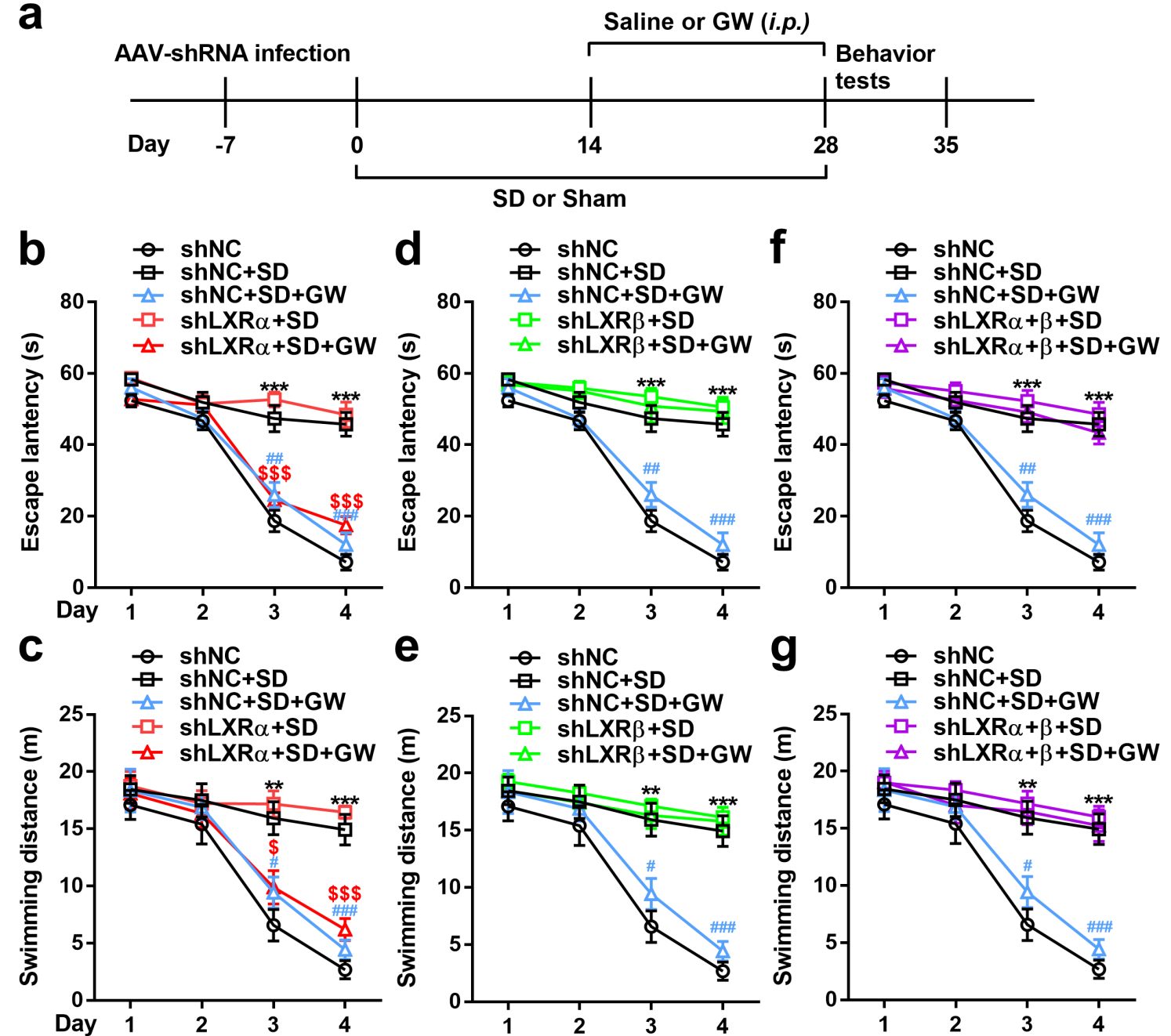

h
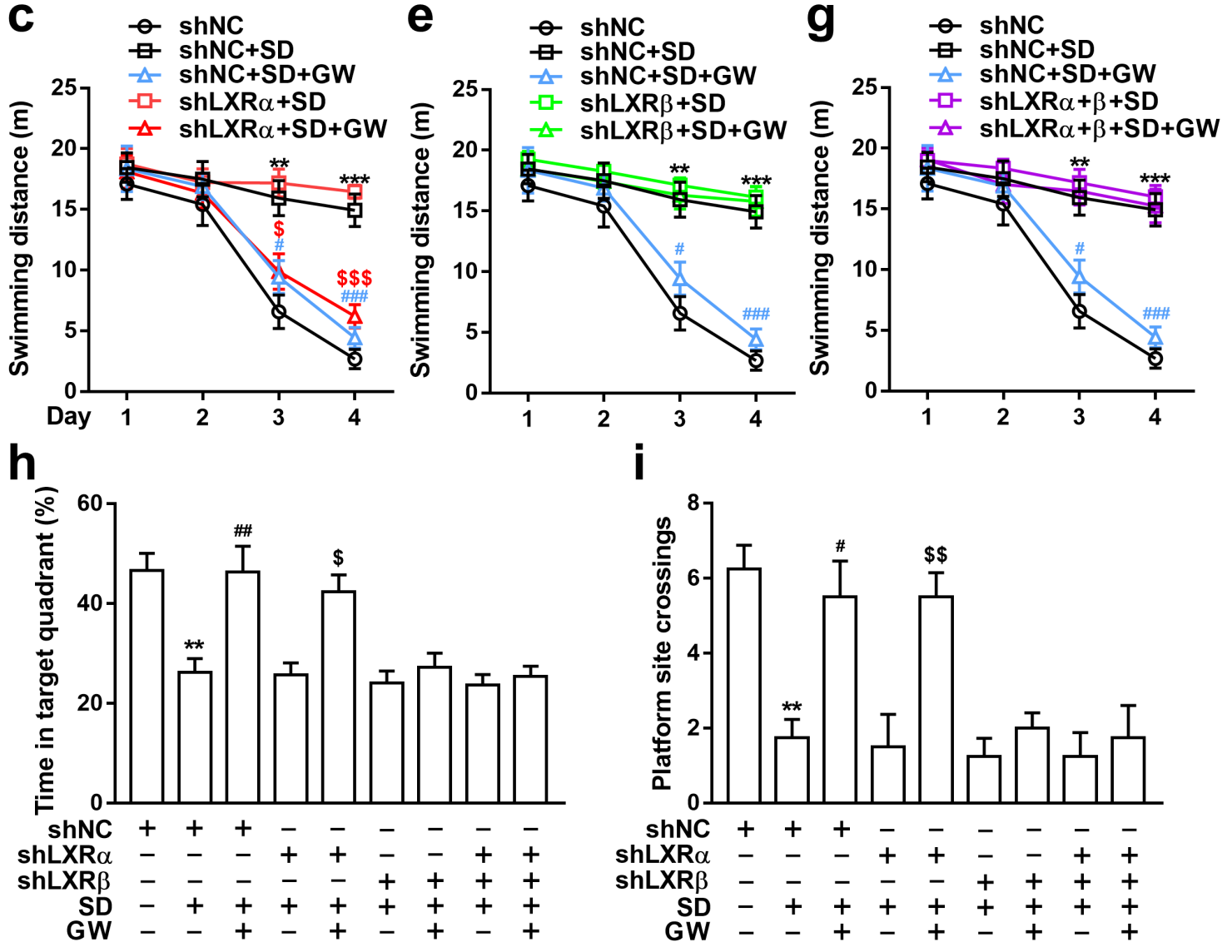

\section{Figure 3}

GW3965 relieved SD-induced cognitive impairment by activating LXRß instead of LXRa. (a) Schematic illustration of the experiment procedure. After AAV-encoding shRNAs infection for $7 \mathrm{~d}$, the mice were deprived of 20 h-sleep a day for $28 \mathrm{~d}$. GW3965 (GW, $10 \mathrm{mg} / \mathrm{kg}$ ) or $0.9 \%$ saline was treated as before. (b-g) The time and distance before reaching the hidden platform during the training phase in MWM test. (b, c) 
induced by SD. (d, e) Downregulation of LXRß by shRNA (shLXRß) abrogated GW3965-mediated learning improvement function. (f, g) Downregulation of LXRa and LXRß also abrogated GW3965-mediated learning improvement function. $(\mathrm{h}, \mathrm{i})$ The memory function of mice in each group was detected during the test stage in MWM test. The knockdown of LXRa did not affect GW in alleviating memory impairment. (h) Percentage of time spent in the target quadrant. (i) Numbers of crossing platform site spent in target quadrant. $\mathrm{n}=4$ in each group, ${ }^{\star \star} \mathrm{p}<0.01$, ${ }^{\star \star *} \mathrm{p}<0.001$, compared with the shNC group; $\# \mathrm{p}<0.05, \# \# \mathrm{p}<$ $0.01, \# \# \# p<0.001$, compared with the shNC + SD group; $\$ p<0.05$,

$$
p<0.01,
$$

$\$ p<0.001$, compared with the shLXRa + SD group. 
a Hoechst

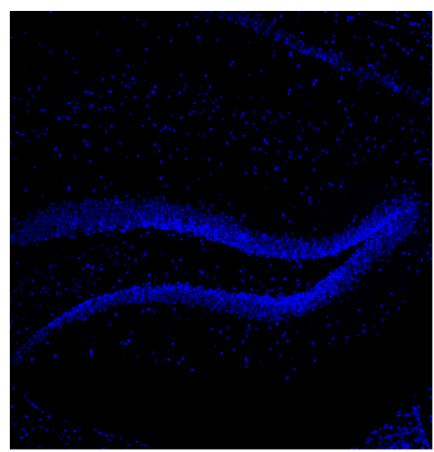

\section{Ctrl}
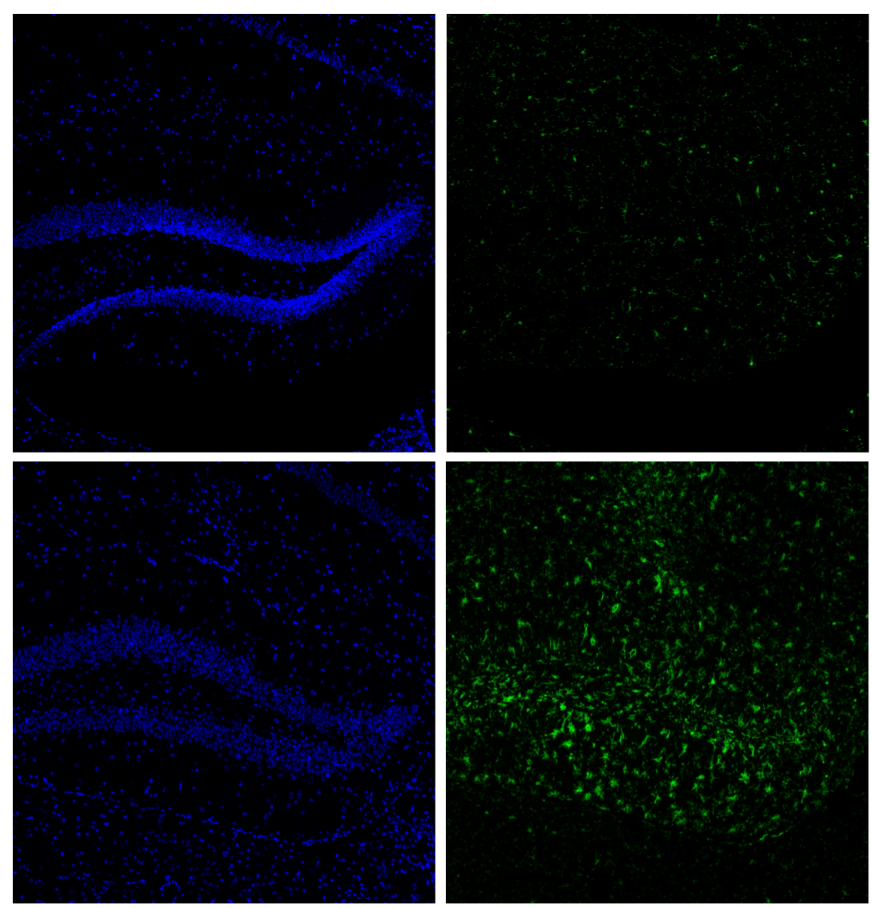

lba-1

Merge
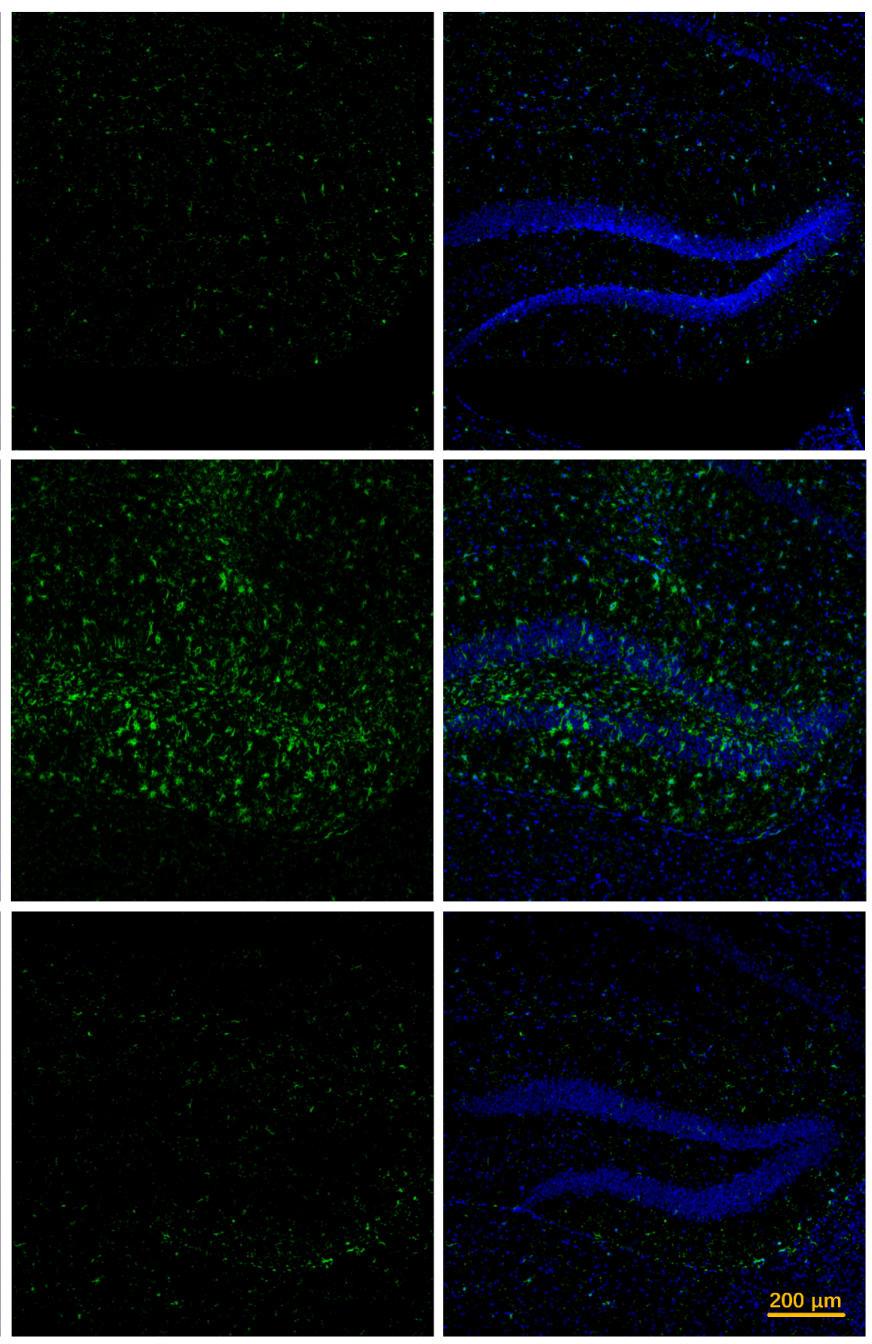

b

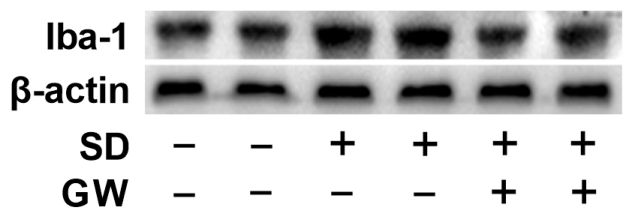

C

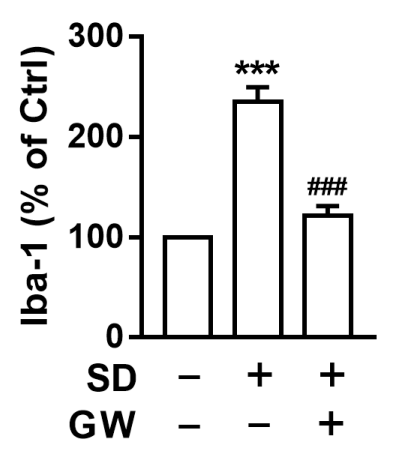

\section{Figure 4}

GW3965 administration suppressed microglia activation in hippocampus induced by SD. (a) The expression changes of Iba-1, a marker of microglia, were measured by immunostaining. Iba-1 was labeled in green and the nucleus was labeled in blue. SD led to an increased expression of Iba-1 in hippocampus, while GW (10 mg/kg) administration suppressed the enhanced expression of Iba-1. (b, c) Representative 
results of Western blot analysis for Iba-1. GW notably suppressed the expression of Iba- 1 in SD mice. $n=$ 6 in each group, ${ }^{\star \star *} p<0.001$, compared with the Ctrl group; \#\#\#p<0.001, compared with the SD group.
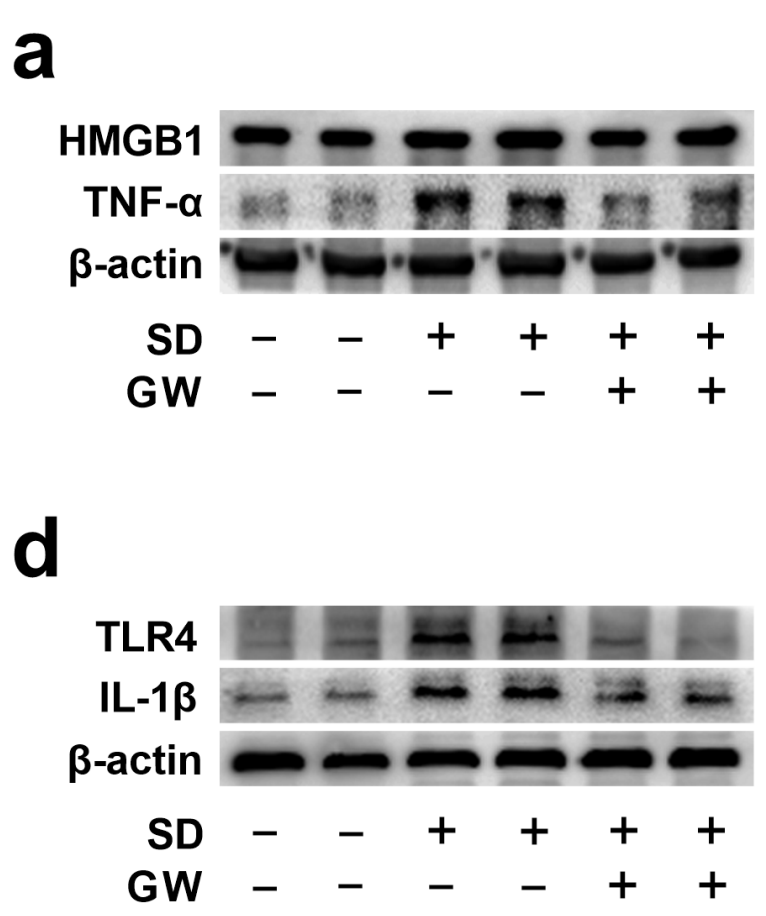

g

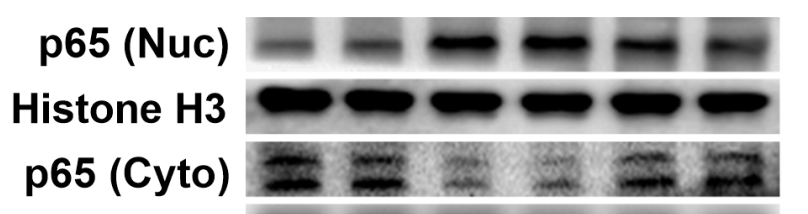

$\beta$-actin

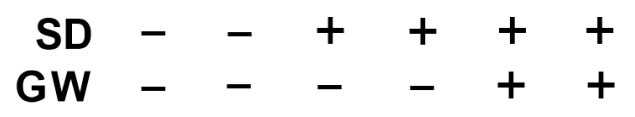

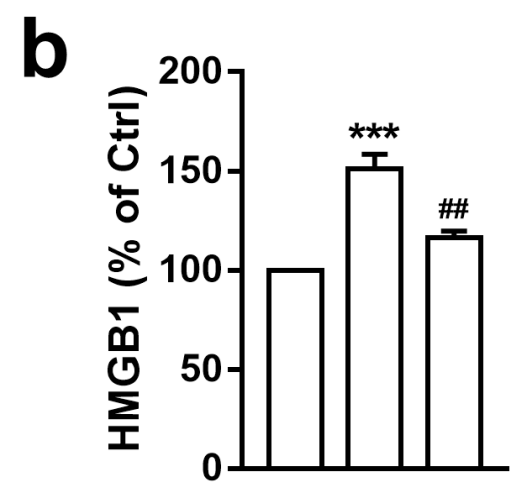

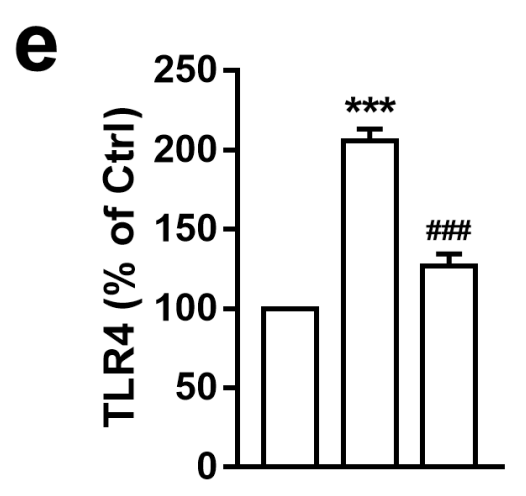

h

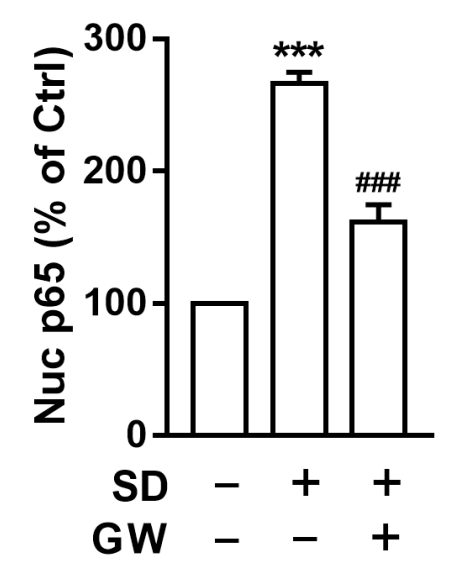

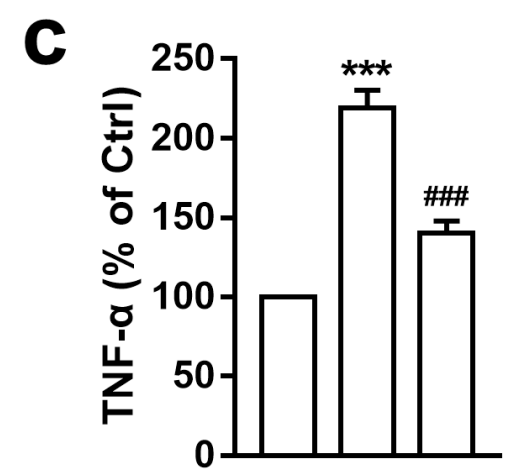
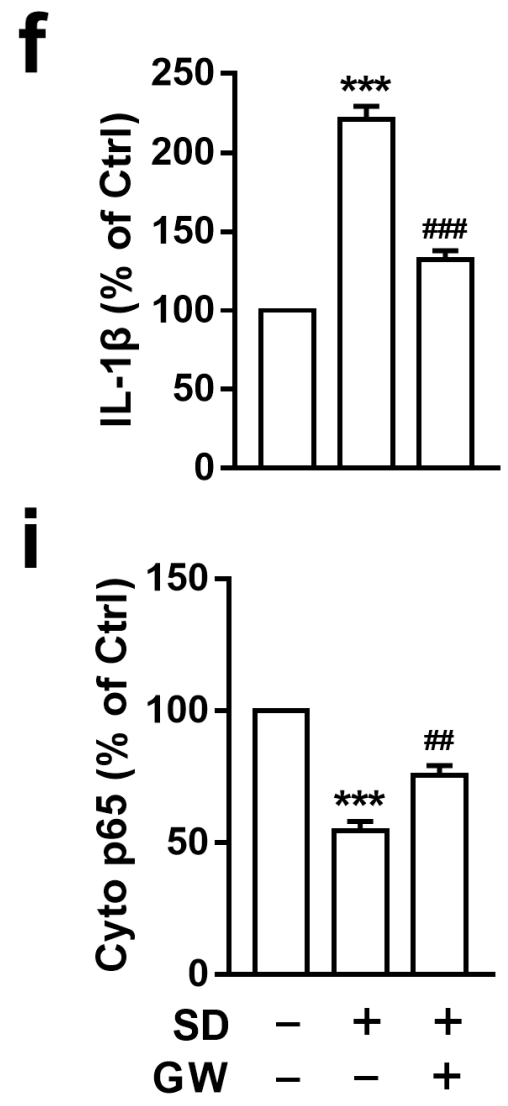

Figure 5

GW3965 treatment inhibited the increased protein levels of HMGB1 pathway in hippocampus upon SD injury. Representative results of Western blot analysis for (a) HMGB1, TNF- $a$, (d) TLR4, IL-1 $\beta$, (g) nuclear (Nuc) and cytoplasmic (Cyto) NF-KB p65. GW treatment (10 mg/kg) inhibited the enhanced expression of (b) HMGB1, (c) TNF-a, (e) TLR4, (f) IL-1 $\beta$ and ( $h$, i) nuclear translocation of NF-KB p65 in hippocampus

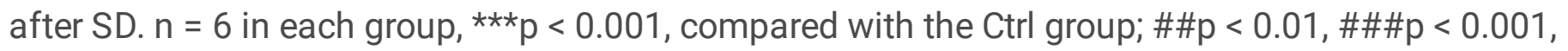
compared with the SD group. 
a

LXR $\beta$

$\beta$-actin

$\operatorname{shNC}++-$ $\operatorname{shLXR} \beta--++$

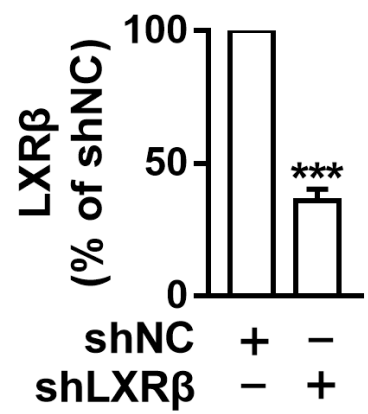

b

\section{HMGB1}

TNF-a

$\beta$-actin
C

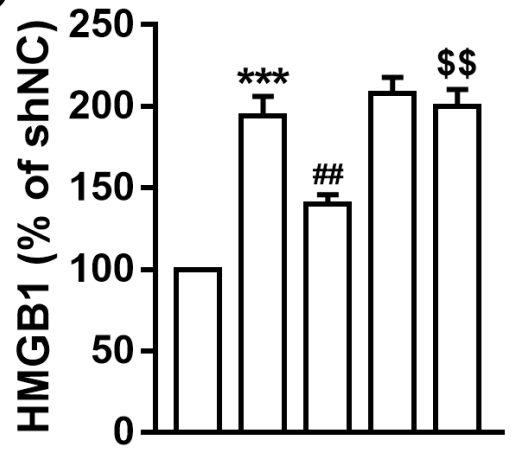

e

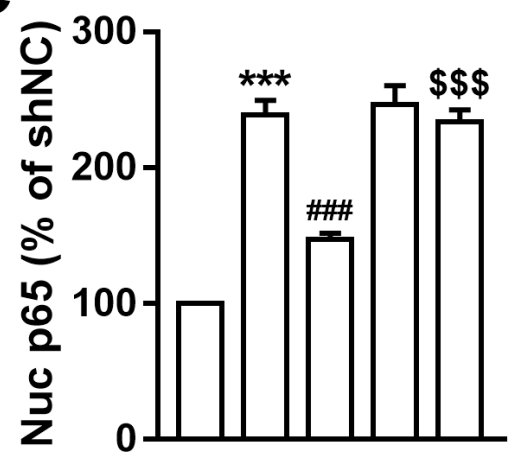

d

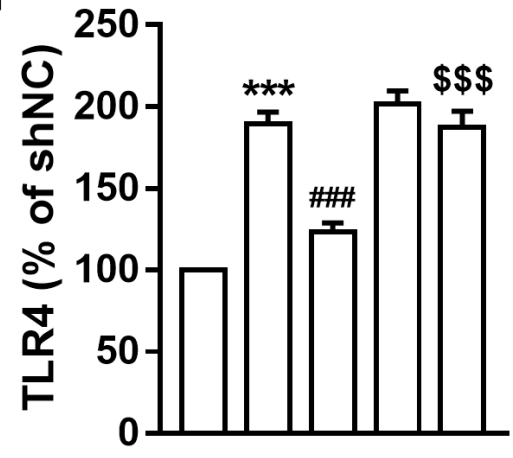

1

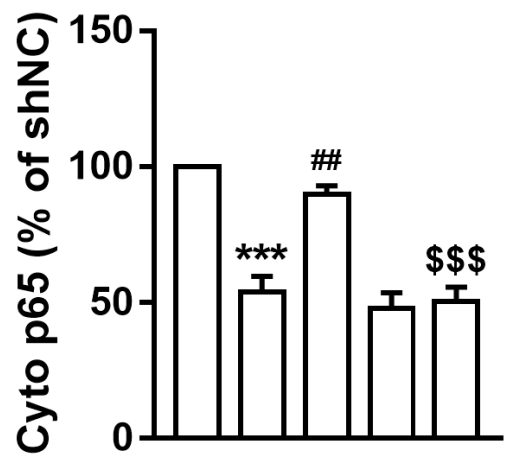

h

TLR4
IL-1 $\beta$

$\beta$-actin

\section{p65 (Nuc)}

Histone H3

p65 (Cyto)

$\beta$-actin

shNC

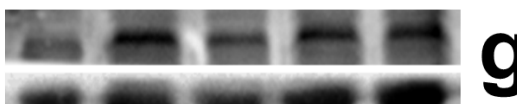

$\operatorname{shLXR\beta }--\infty++$

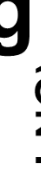

$\bigcup_{0}^{250}$
$\sum_{\frac{1}{0}} 200$
$\frac{0}{0} 150$

○ 100

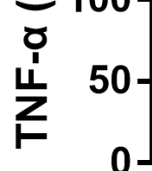

shNC +++-

$\operatorname{shLXR\beta }--++$

LPS+ATP -++++ LPS+ATP -++++

GW - - + - +

$\mathrm{GW}--+-+$

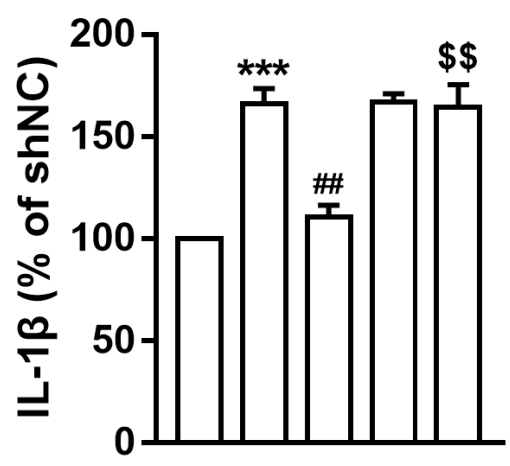

$\operatorname{shNC}+++-$

ShLXRß - -++

LPS+ATP -++++

GW - -+-+

Figure 6

GW3965 suppressed HMGB1 pathway in the murine N9 microglia activated by LPS and ATP through activating LXRß. (a) After 3 d-infection of shLXRß adeno-associated virus (AAV), the level of LXR 3 was

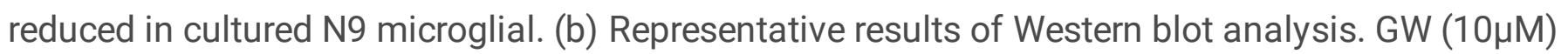
treatment inhibited the elevated levels of (c) HMGB1, (d) TLR4, (g) TNF- $a$, (h) IL-1 $\beta$ and (e, f) nuclear translocation of NF-KB p65 upon LPS/ATP stimulation. Knockdown of LXR $\beta$ expression prevented GW from inhibiting HMGB1 pathway. $\mathrm{n}=6$ in each group, $* \star \star p ~<0.001$, compared with the shNC group; \#\#p< Loading [MathJax]/jax/output/CommonHTML/jax.js 


$$
p<0.01,
$$

$\$ p<0.001$, compared with the shNC + LPS/ATP + GW group.

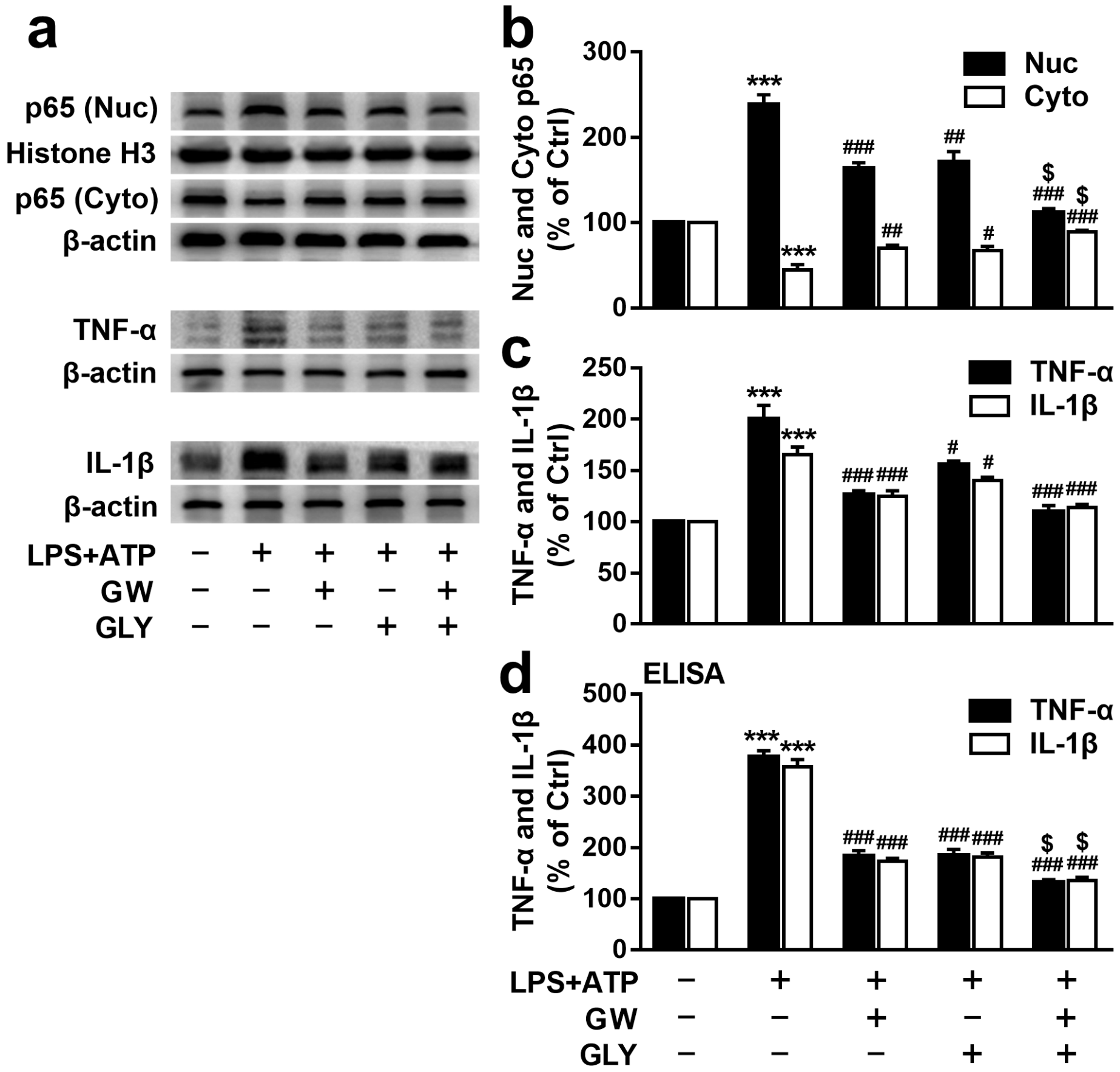

Figure 7

HMGB1 inhibitor GLY synergistic promoted GW3965-mediated effects in inhibiting inflammatory responses induced by LPS and ATP in murine N9 microglia. (a) The expression levels of nuclear (Nuc) and cytoplasmic (Cyto) NF-KB p65, TNF- $a$ and IL-1 $\beta$ were determined by Western blot. HMGB1 inhibitor GLY promoted the inhibitory effect of GW on (b) nuclear translocation of NF-KB p65, (c) the expression of Loading [MathJax]/jax/output/CommonHTML/jax.js IL-1 $\beta$ in supernatants from each group were evaluated by 
a

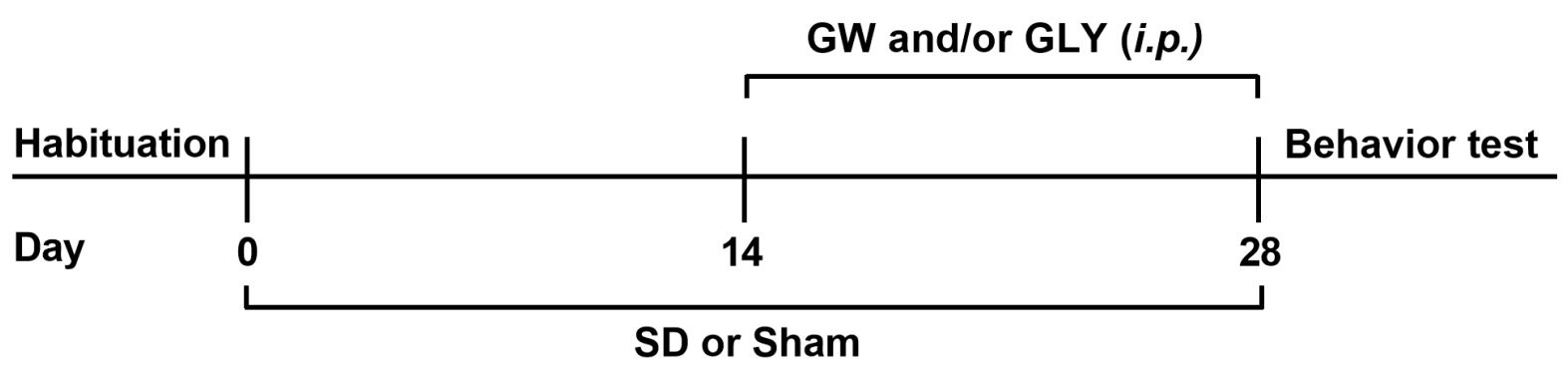

b

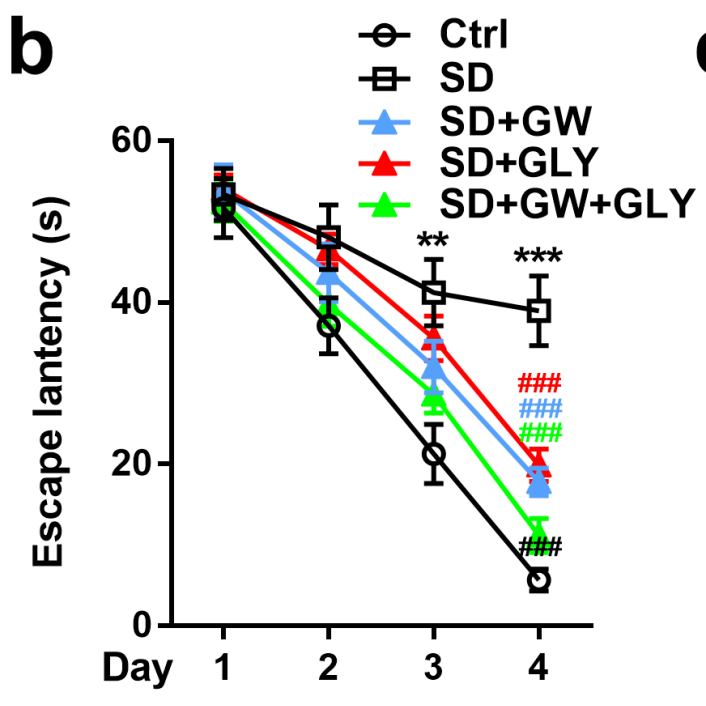

d
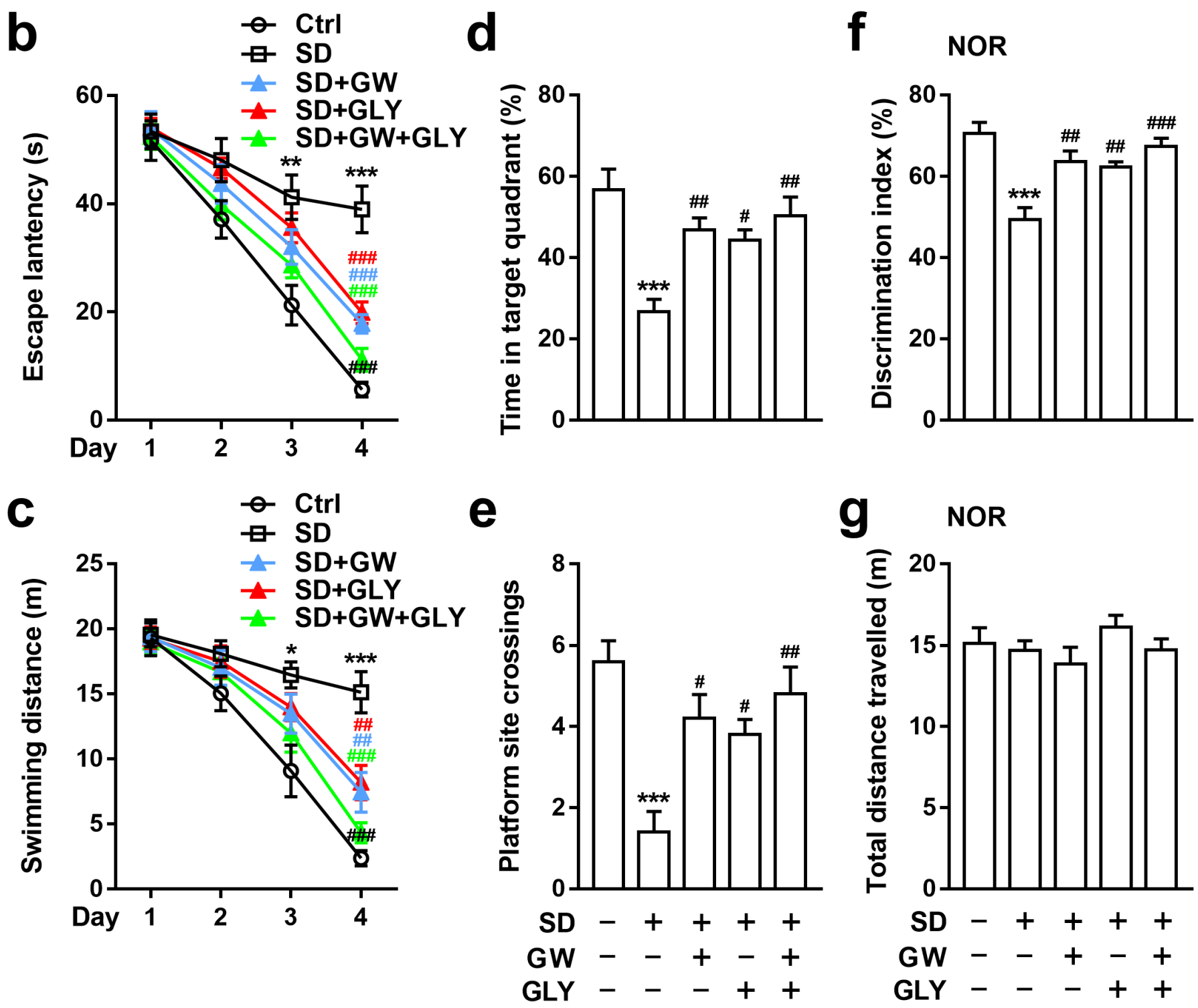

Figure 8

Inhibition of HMGB1 facilitated GW3965-mediated improvement of cognitive impairment induced by SD. (a) Schematic illustration of the experiment procedure. GLY $(20 \mathrm{mg} / \mathrm{kg})$ and/or GW $(10 \mathrm{mg} / \mathrm{kg})$ was Loading [MathJax]/jax/output/CommonHTML/jax.js $14 \mathrm{~d}$ after SD, for $14 \mathrm{~d}$ consecutively. (b-f) GLY could alleviate 
the cognitive dysfunction induced by SD, and the effect tends to be enhanced when combined with GW. (b) Mean daily escape latencies. (c) Total swimming distance during the learning phase. (d) Percentage of time spent in the target quadrant. (e) Numbers of crossing platform site spent in target quadrant. (f) Discrimination index toward a novel object. (g) There was no significant difference in the locomotor ability of mice in each group. $\mathrm{n}=5$ in each group, ${ }^{\star \star \star} \mathrm{p}<0.001$, compared with the Ctrl group; $\# p<0.05$, $\# \# p<0.01$, \#\#\#p <0.001, compared with the SD group.

\section{Supplementary Files}

This is a list of supplementary files associated with this preprint. Click to download.

- extendeddatafigure1.tif

- extendeddatafigure2.tif

- extendeddatafigure3.tif

- extendeddatafigure4.tif 